\title{
Interplanetary flux rope ejected from an X-ray bright point
}

\section{The smallest magnetic cloud source-region ever observed}

\author{
C. H. Mandrini ${ }^{1}$, S. Pohjolainen ${ }^{2}$, S. Dasso ${ }^{1,3}$, L. M. Green ${ }^{4}$, P. Démoulin ${ }^{5}$, L. van Driel-Gesztelyi ${ }^{5,6,7}$, \\ C. Copperwheat ${ }^{6}$, and C. Foley ${ }^{6}$ \\ 1 Instituto de Astronomía y Física del Espacio, CONICET-UBA, CC. 67, suc. 28, 1428 Buenos Aires, Argentina \\ e-mail: mandrini@iafe.uba.ar,sdasso@iafe.uba.ar* \\ 2 Tuorla Observatory/VISPA, University of Turku, 21500 Piikkiö, Finland \\ e-mail: silpoh@utu.fi \\ ${ }^{3}$ Departamento de Física, Facultad de Ciencias Exactas y Naturales, UBA, Buenos Aires, Argentina \\ e-mail: dasso@df.uba.ar \\ 4 The Faulkes Telescope Project, Cardiff University, 5 the Parade, Cardiff, CF24 3YB, UK \\ e-mail: lucie.green@faulkes-telescope.com \\ 5 Observatoire de Paris, LESIA, UMR 8109 (CNRS), 92195 Meudon, France \\ e-mail: [Pascal.Demoulin; Lidia.vanDriel]@obspm.fr \\ ${ }^{6}$ Mullard Space Science Laboratory, University College London, Holmbury St. Mary, Dorking, Surrey RH5 6NT, UK \\ e-mail: [lvdg;cmc;caf] @mssl.ucl.ac.uk \\ 7 Konkoly Observatory, Pf. 67, 1525 Budapest, Hungary \\ e-mail: vandriel@konkoly.hu
}

Received 13 April 2004 / Accepted 5 January 2005

\begin{abstract}
Using multi-instrument and multi-wavelength observations (SOHO/MDI and EIT, TRACE and Yohkoh/SXT), as well as computing the coronal magnetic field of a tiny bipole combined with modelling of Wind in situ data, we provide evidences for the smallest event ever observed which links a sigmoid eruption to an interplanetary magnetic cloud (MC). The tiny bipole, which was observed very close to the solar disc centre, had a factor one hundred less flux than a classical active region (AR). In the corona it had a sigmoidal structure, observed mainly in EUV, and we found a very high level of nonpotentiality in the modelled magnetic field, 10 times higher than we have ever found in any AR. From May 11, 1998, and until its disappearance, the sigmoid underwent three intense impulsive events. The largest of these events had extended EUV dimmings and a cusp. The Wind spacecraft detected 4.5 days later one of the smallest MC ever identified (about a factor one hundred times less magnetic flux in the axial component than that of an average MC). The link between this last eruption and the interplanetary magnetic cloud is supported by several pieces of evidence: good timing, same coronal loop and MC orientation, same magnetic field direction and magnetic helicity sign in the coronal loops and in the MC. We further quantify this link by estimating the magnetic flux (measured in the dimming regions and in the MC) and the magnetic helicity (pre- to post-event change in the solar corona and helicity content of the MC). Within the uncertainties, both magnetic fluxes and helicities are in reasonable agreement, which brings further evidences of their link. These observations show that the ejections of tiny magnetic flux ropes are indeed possible and put new constraints on CME models.
\end{abstract}

Key words. Sun: magnetic fields - Sun: corona

\section{Introduction}

Coronal mass ejections (CMEs) are commonly thought to be phenomena that involve a large-scale reconfiguration of the solar corona, accompanied by significant disturbances in the solar wind. However, there is a small subset of events, the so-called narrow CMEs, which do not conform to this picture. Narrow CMEs are arbitrarily defined as events whose apparent width

* C.H.M. and S.D. are members of the Carrera del Investigador Científico (CONICET). is $15^{0}$ or less and are usually referred to as rays, spikes, fans, etc. (Munro \& Sime 1985; Howard et al. 1985). Within this category there seems to be two types of ejections: the ones that exhibit no interior structure and have jet-like characteristics, and those that are structured and do not show any obvious difference with large CMEs (Harrison et al. 2001; Gilbert et al. 2001; Dobrzycka et al. 2003). Coronal jets and CMEs are entirely different phenomena. Jets are thought to be the result of reconnection between open and closed field lines where the plasma can be strongly heated and accelerated (Shibata et al. 1992). 
CMEs, on the other hand, are often attributed to the release of magnetic stress stored in a twisted flux tube or in a sheared arcade, and they involve the ejection of part of the magnetic flux and twist of the source region into the interplanetary space (see e.g. Mikic \& Linker 1994; Gibson \& Low 1998; Antiochos et al. 1999; Lin \& Forbes 2000).

Strongly non-potential bipolar magnetic configurations usually have coronal loops that have a sigmoidal shape when viewed from above. Sigmoids are preferentially observed in soft X-rays (Sterling \& Hudson 1997). However, in some cases, sigmoids do appear in EUV images (see e.g. Sterling et al. 2000). Rust \& Kumar (1996) found that many large transient brightenings in X-rays associated with CMEs were S-shaped. Though the sigmoid - CME connection is still statistically ambiguous (Canfield et al. 1999; Glover et al. 2000), it has been suggested that the magnetic helicity content in S-shaped magnetic configurations may reach a threshold leading to instability and eruption (van Driel-Gesztelyi et al. 2000; Török \& Kliem 2003 and references therein). In the same way as CMEs can go from large-scale to very narrow events, the scale of sigmoidal structures can range from interconnecting loop size to the size of a typical AR (e.g. Siarkowski et al. 2002).

CMEs appear in the interplanetary medium as interplanetary CMEs (ICMEs). A subset of these ICMEs, called magnetic clouds (MCs), has well defined characteristics: a coherent rotation of the magnetic field vector, an enhanced field strength, as well as, a proton temperature lower than in the surrounding solar wind (Burlaga et al. 1981). This subset has been thoroughly studied. There is increasing evidence that the helicity sign of MCs matches that of their solar source region (Bothmer \& Schwenn 1994; Rust 1994; Marubashi 1997; Yurchyshyn et al. 2001).

Most of the MC studies have so far focused on large scale events which are observed during a few days (see e.g. Lepping et al. 1990; Zhao et al. 2001), but recently much smaller interplanetary flux ropes have also been detected (Shimazu \& Marubashi 2000). The statistical distributions of the MC main physical characteristics, such as their spatial extension, magnetic flux and helicity, are presently poorly known.

Recognizing the broad spatial and temporal scales in CME source regions, CMEs and ICMEs, we can ask the following questions:

1. What is the smallest magnetic bipole that can produce a CME and launch a twisted flux tube into the interplanetary space?

2. What is the smallest magnetic flux tube ejected in a CME that can reach the Earth and still be recognizable as a MC?

During a survey of X-ray bright points that showed enhanced emission at $\mathrm{cm}$ - and mm-wavelengths (Pohjolainen 2000), we observed an isolated radio bright point near the center of the disc on May 11, 1998. We found multi-wavelength evidence of its eruptive nature, such as elongated sigmoidal loops which later disappeared, EUV dimmings and cusp formation (in the largest event). Later, the ejected plasma and magnetic field were observed in the interplanetary space as a small MC. The bright point location is shown in soft X-rays just before its largest eruption, together with a full disc magnetogram, in

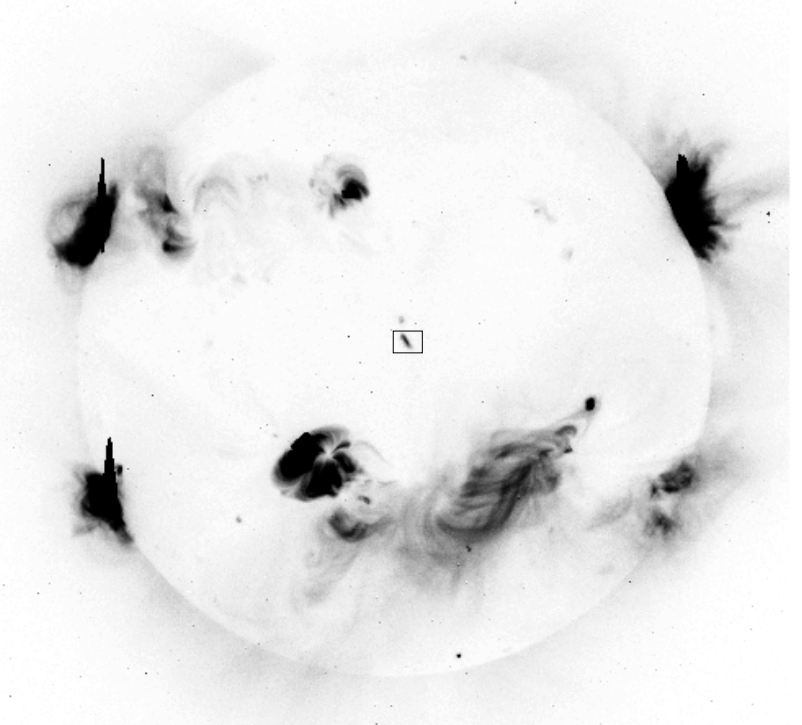

11 May 1998 Yohkoh SXT 07:50:31 UT

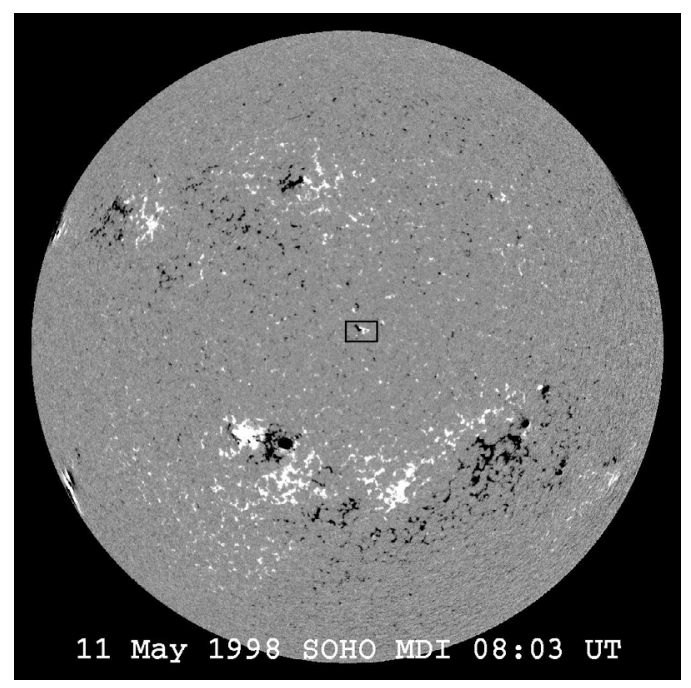

Fig. 1. SXT full disc image at the time of maximum extension of the sigmoidal X-ray bright point (top) and the closest in time MDI magnetogram (bottom). The bright point is associated to a very small AR located at disc centre (both shown within the tiny boxes), which is very far from other ARs.

Fig. 1. It was well isolated from ARs present on the Sun. It was also located almost at the disc centre in an ideal position for any ejecta to reach the Earth.

We first describe, in Sect. 2, the global evolution of the small bipolar AR at the photospheric level (magnetic field) and in the corona (EUV and X-rays) from its birth to its complete decay. Then, the coronal eruptions are analyzed in Sect. 3. We describe the evidence for the ejections and quantify the amount of magnetic flux and helicity involved. We concentrate in the study of the largest event (in terms of time integrated X-ray flux and EUV dimming extension). In Sect. 4, we analyze the interplanetary data plausibly associated with this coronal ejection. In particular, we derive the same physical quantities as on the Sun. In Sect. 5, we link the events observed in the corona 
and the interplanetary space. Finally, in Sect. 6, we conclude that the ejection differs only in scale from other CME events, being the same true for its associated MC. We briefly discuss our results in the context of CME models.

\section{Evolution of the small AR during its full life-time}

\subsection{Total magnetic flux}

We follow the evolution of the photospheric magnetic field using the data from the Michelson Doppler Imager (MDI, Scherrer et al. 1995) on board the Solar and Heliospheric Observatory (SOHO). The magnetograms are computed from the data taken in five narrowband $(94 \mathrm{~m} \AA$ ) filtergrams at different positions along the Ni I $6767.8 \AA$ absorption line, formed in the mid-photosphere. The pixel size of the full disc magnetograms is 1.98 arcsec. MDI returns the longitudinal magnetic flux density averaged over the pixel field of view, and from this the flux within a required area can be computed.

To measure the magnetic flux, a polygonal contour, defined by eye, is fitted around the bipole and the flux is summed within it. In this way we minimize the contribution of the background field and of the noise in low field regions $(B \leq 10 \mathrm{G})$. Measurements in the surrounding quiet Sun regions of the same size give a flux below $3 \%$ of the bipole maximum flux. We correct all the magnetic field measurements following the results of Berger \& Lites (2003), who analyzed co-temporal and cospatial MDI and Advanced Stokes Polarimeter (ASP) data. All the MDI measurements are below $1200 \mathrm{G}$, so the relationship between the two instruments is linear, and we simply multiply the magnetic field measurements by a factor 1.45 (since Berger $\&$ Lites (2003) have found $B_{\mathrm{MDI}} \approx 0.69 B_{\mathrm{ASP}}$ ). According to our measurements, the magnetic flux of the bipole at peak evolution (average between positive and absolute value of the negative fluxes, corrected for the flux underestimation by MDI) was $3.2 \times 10^{20} \mathrm{Mx}$. This peak flux puts this bipole into the "small active region" category that are defined to be in a flux range of $1-50 \times 10^{20} \mathrm{Mx}$ (Schrijver \& Zwaan 2000). These small ARs are frequently spotless or at most contain pores. In the MDI white-light images this bipole appeared spotless and even poreless during its entire existence.

The lifetime of ARs is roughly proportional to their flux content at maximum development. Flux decay rate is between $1-2 \times 10^{20} \mathrm{Mx} /$ day (Golub 1980; Harvey 1993, respectively). For an AR having a magnetic flux of $3.2 \times 10^{20} \mathrm{Mx}$ at peak evolution, the entire lifetime from birth to disappearance is short since the bipole is expected to exist for about 1.5-3 days. In this particular case, we indeed observed the bipole for $64 \mathrm{~h}$ (2.7 days, see Fig. 2).

\subsection{Total $X$-ray flux}

We analyze the evolution of the X-ray flux of this small spotless AR with the Yohkoh/Soft X-ray Telescope (SXT, Tsuneta et al. 1991). The full-disc images have a pixel size of 5 arcsec. We used the software SXT_FLUX with a fixed temperature of 2 MK for this AR, which we obtained from the temperature
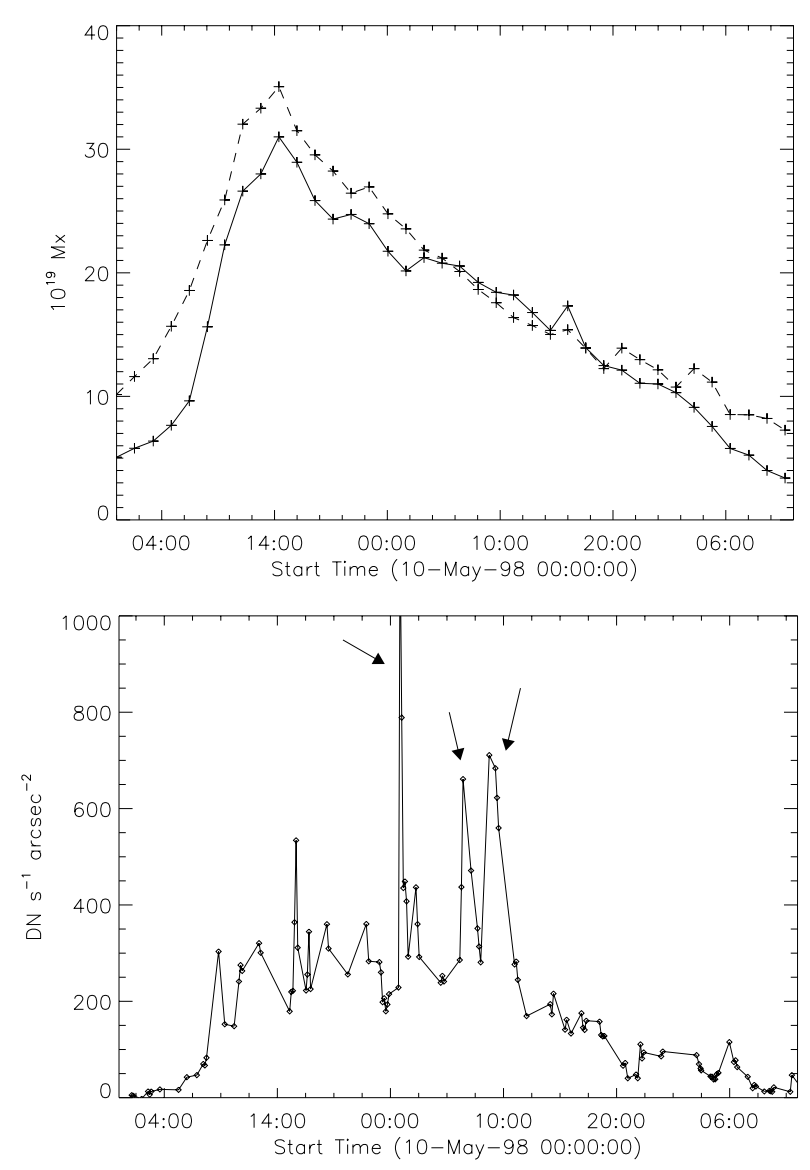

Fig. 2. Magnetic (top) and X-ray flux evolution (bottom) from the emergence to the disappearance of the small active region. The positive (negative) magnetic flux is shown with continuous (dashed) line. There are three main bursts in X-rays (marked with arrows in the bottom figure), which all occurred on May 11: the first at 01:00 UT, the second at $\sim 07: 00 \mathrm{UT}$, and the third at $\sim 09: 00 \mathrm{UT}$.

analysis using the thin aluminum (Al1) and the Dagwood filters (Al-Mg).

Outside flaring times (i.e. outside $\mathrm{X}$-ray peaks), the $\mathrm{X}$-ray flux is correlated in time with the total magnetic flux (Fig. 2). This is in agreement with several previous studies. Harvey (1997) showed that the total magnetic flux and the intensity increase of an X-ray bright point, related to an emerging bipole, were almost linearly proportional. Fisher et al. (1998) found that the X-ray luminosity $\left(L_{\mathrm{X}}\right)$ of solar ARs is best correlated with the total unsigned magnetic flux $\left(\Phi_{\text {tot }}\right)$ of the region. The $L_{X}-\Phi_{\text {tot }}$ relationship was found to follow a power-law with an exponent of 1.19. Pevtsov et al. (2003) extended this result to a broad range of structures on the Sun (quiet Sun, X-ray bright points, active regions, and integrated solar disc) and to active stars (dwarf and pre-main-sequence).

However, there appear to be differences between the shapes of the magnetic and X-ray flux evolution curves. Magnetic flux peaks about eleven hours after the start of emergence, then decreases steadily. The X-ray flux, outside flaring times, reaches maximum ten hours later than the magnetic flux, which may be regarded as the storage time of the free magnetic energy. 


\subsection{X-ray bursts}

The soft X-ray light curve presents several peaks (Fig. 2). The first small flare event is observed as early as about two hours after the appearance of the XBP, and about eight hours after the start of the emergence of the bipole on 10 May. The most intense events are observed during May 11 (Fig. 2). The exact times of the X-ray bursts are difficult to define because of the low temporal cadence of SXT full-disc images.

The first event started between the images taken at 00:42 UT and 00:51 UT. At 01:08 UT, the SXT flux had gone down to the pre-event level, so its maximum duration was $26 \mathrm{~min}$. This first X-ray burst was also observed by the Nobeyama Radioheliograph (Nishio et al. 1994) at $17 \mathrm{GHz}$ (1 s time cadence). From the constructed radio flux curve, the burst began at 00:45 UT, peaked at 00:48 UT and ended at 00:58 UT, having a total duration of $13 \mathrm{~min}$.

Two close in time X-ray bursts followed the first one. The second event occurred between 06:00 UT and 08:00 UT, while the third one started between SXT full-disc images at 08:03 UT and 08:44 UT and had a maximum between 08:03 UT and 09:16 UT. The post-maximum evolution of this event was imaged with a higher temporal cadence and we can define its end as 11:00 UT. Then, its duration was of about $3 \mathrm{~h}$. Unfortunately, this third burst occurred too late for Nobeyama and was not imaged in radio.

\subsection{Indirect evidence of magnetic twist}

The orientation of the magnetic bipole was observed to change with time (Fig. 3), the axis joining both polarities rotated. Such rotation could come from the emergence of a non-planar magnetic flux tube (as studied for ARs, López Fuentes et al. 2003). However, in the present case most of the rotation is likely to be apparent and probably due to the emergence of a strongly twisted flux tube, as discussed below.

The presence of twist in an emerging flux tube can influence the distribution of the line-of-sight magnetic field in such a way that, for a negative twist, the preceding polarity extends along the northern boundary of the following polarity (forming an elongated "magnetic tongue"). This has been proposed by López Fuentes et al. (2000, see their Fig. 5 and corresponding text). In the two first magnetograms of Fig. 3, only the upper part of the flux tube (i.e. above the flux tube axis) has emerged across the photosphere. At this time, mostly the azimuthal component (associated to the twist) is contributing to the longitudinal field (which mainly corresponds to the vertical component because of the near central disc position).

As the emergence proceeds, the axial magnetic component of the flux tube contributes more to the vertical photospheric component, forming the classical separating polarities in magnetic bipoles (see magnetograms at 12:47 and 19:11 UT in Fig. 3). At these times, the photospheric magnetic distribution is formed by two separating polarities and by two magnetic tongues. As emergence continues, the magnetic tongues retract (see magnetograms from 1:39 UT to 12:51 UT on May 11) because (i) the azimuthal component is less important closer to the central part of the flux tube and (ii) it has a smaller vertical projection in the legs of the flux tube.

In the case of a strongly twisted flux tube, this evolution leads to an apparent rotation of the photospheric magnetic polarities by about 90 degrees. The orientation (or chirality) of the magnetic tongues is directly linked to the sign of the twist (López Fuentes et al. 2000). Therefore, we conclude from the evolution of the longitudinal field distribution that the emerging flux tube had a strong negative twist.

\subsection{Spatial distribution of the EUV flux}

The complete evolution of the EUV emission of this small AR is best covered by the observations obtained with the SOHO/Extreme-Ultraviolet Imaging Telescope (EIT, Delaboudiniere et al. 1995). EIT observes the full Sun regularly with four different filters and with a pixel size of 2.6 arcsec (Fig. 4). Globally the EUV emission followed the evolution of the photospheric magnetic field (Fig. 3); in particular, the emission extends and rotates in parallel to the photospheric "boundary conditions".

On top of the previously described global behavior, there is also a specific evolution of the EUV emission. This evolution is not directly linked to the co-temporal photospheric magnetic field, but rather to the magnetic stress accumulation in the corona, and later to the global magnetic instability.

The small AR was first observed as a bright point in EUV on May 10, but soon after distinct elongated loops began to form (Fig. 4). By 19:10 UT on May 10, the emission resembles a sigmoidal structure. This shape is also present in the EIT images at 00:35 UT, 00:53 UT and 8:31 UT on May 11 (Fig. 5). The loops are better seen with the Transition Region and Coronal Explorer data (TRACE, Handy et al. 1999) since TRACE has $\approx 5$ times better spatial resolution than EIT (but TRACE observations were unfortunately limited to the time around 00:38 UT on May 11, and so do not make it possible to follow the AR evolution). A central elongated loop is present connecting the positive and negative polarities of the small AR (Fig. 8, top). J-shaped loops, as those expected in eruptive twisted bipolar configurations (Démoulin et al. 1996), are seen on the northern part. Elongated sigmoidal and/or J-shaped structures are considered to be signatures of eruptive phenomena. In the view of Rust \& Kumar (1996) and Pevtsov et al. (1996) these structures are erupting loops or ends of the erupting flux rope (in this way we have named the loops in Fig. 5). However, Démoulin et al. (1996) and Titov \& Démoulin (1999) suggested by computing the magnetic field topoloqy (i.e. the quasi-separatrix layers and the separatrices associated to the bald patches, respectively) in a twisted magnetic configuration that the elongated sigmoids are not the erupting loops but enhanced current density layers in which the main energy release occurs. The results of these topological studies are supported by the recent numerical simulations of Fan \& Gibson (2003), Gibson et al. (2004) and Kliem et al. (2004). For the observations described in the present paper both interpretations are plausible, since in both cases a sigmoidal or J-shaped structure is an indicator of a twisted flux rope that tends to instability. 


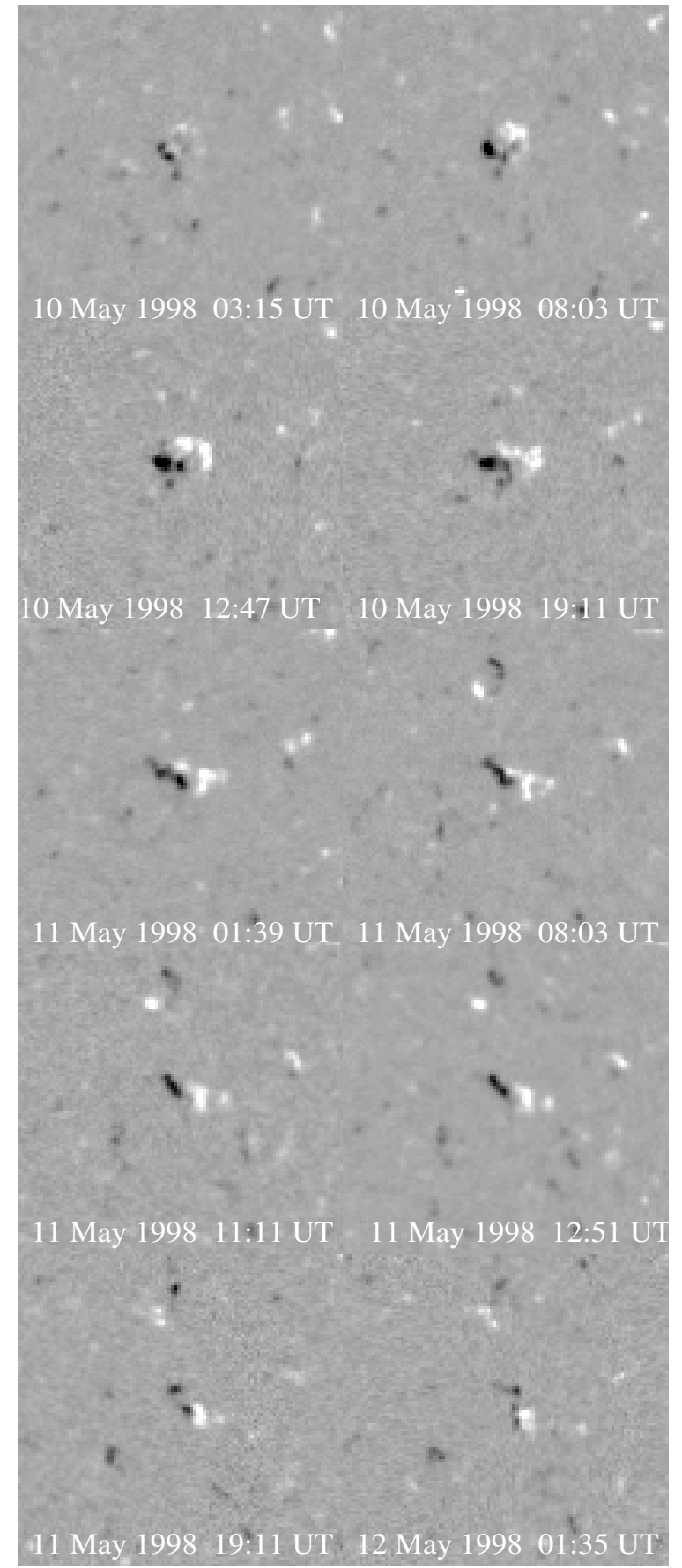

Fig. 3. Photospheric magnetograms from SOHO/MDI showing the full evolution of the bright point region from May 10 to 12, 1998. The field of view is $200 \times 200 \operatorname{arcsec}(1.98$ arcsec per pixel), the maps have been coaligned and derotated to central meridian position (11 May 04:15 UT).

From previous studies (see Introduction), we expect to see the sigmoidal loops best defined at higher temperatures than those observed by EIT and TRACE, like in SXT images. Unluckily these very small events were only imaged by SXT in full-disc images in which the spatial resolution is low compared to that of the EUV instruments. In X-rays mainly an elongated structure is seen (Figs. 1 and 13, top).

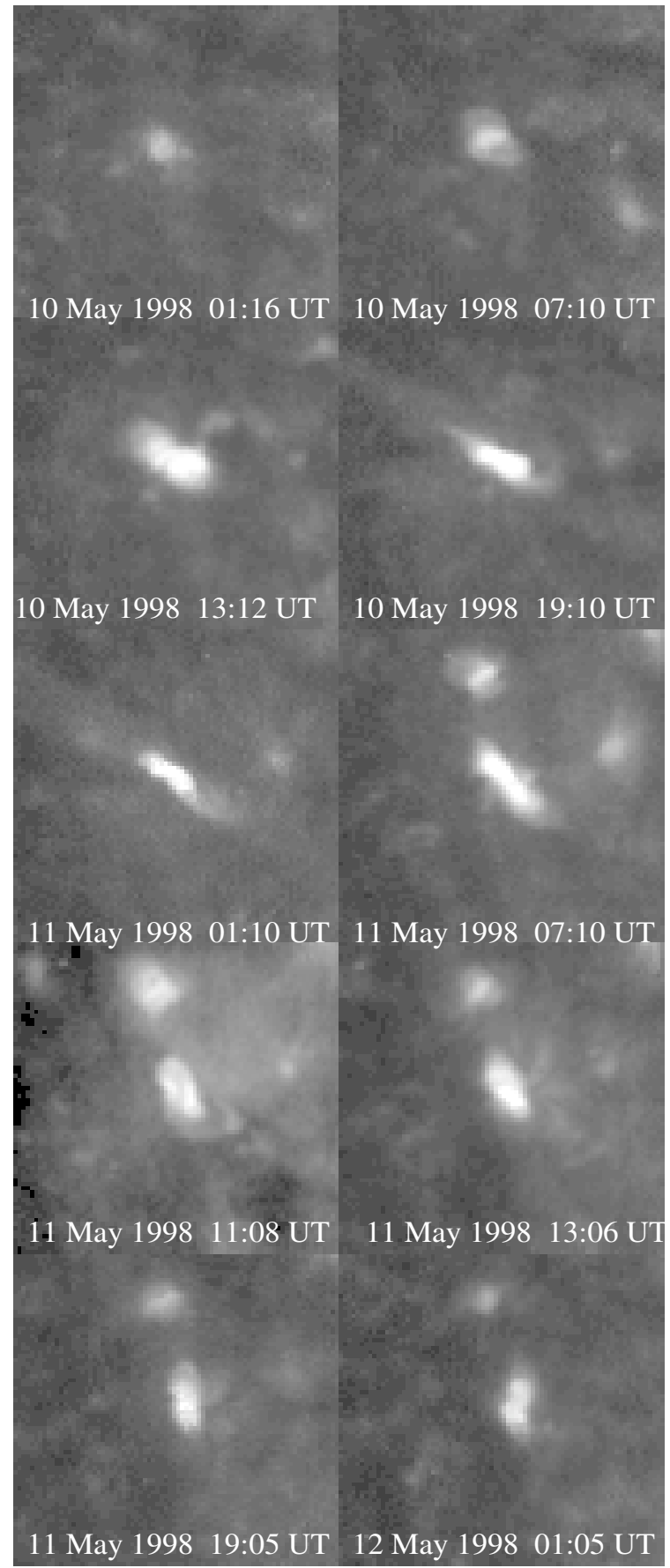

Fig. 4. Coronal evolution as seen in EUV with the SOHO/EIT (same field of view and treatment as in Fig. 3, 2.62 arcsec per pixel). All the images shown here are taken with the $284 \AA$ filter, except the one on 11 May at 11:08 UT where the wavelength is $195 \AA$.

\subsection{EUV events}

A small event can be observed in EIT images at $\approx 01: 00$ UT on 11 May; the $284 \AA$ image at 01:10 UT in Fig. 4 shows the corresponding weak "flare" loops. This event is related to the first X-ray burst starting after 00:42 UT in the SXT curve (see Fig. 2 and Sect. 2.3). During the event there is a shortening of the emitting region and the formation of a dimming region (Fig. 5, top). Next, the second X-ray burst is associated to the weakest 


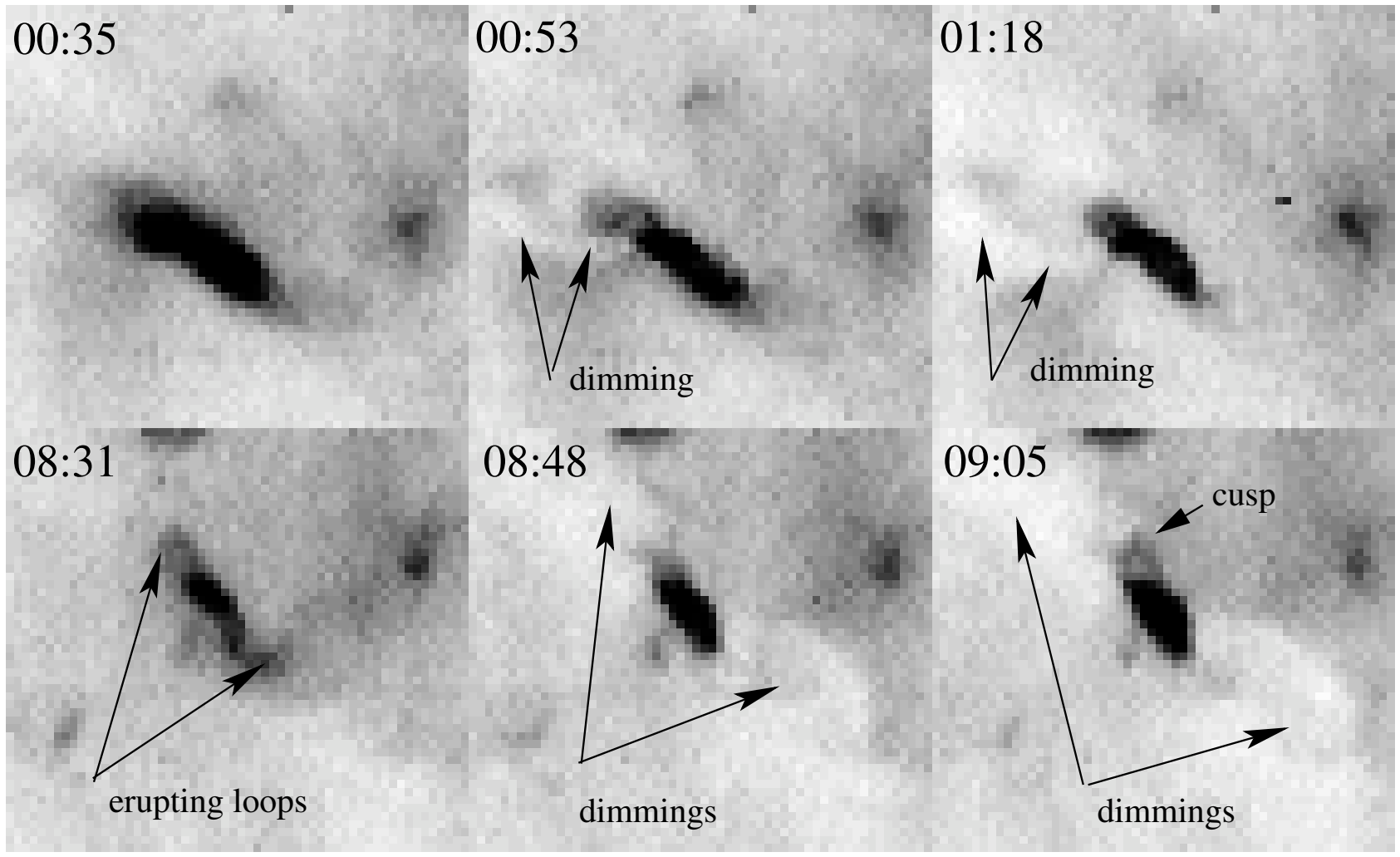

Fig. 5. EIT $195 \AA$ images in contrast-enhanced reversed colors (enhanced emission in black, reduced in white) of the bright point during the first and third X-ray bursts indicated in Fig. 2, bottom. The field of view is $150 \times 135$ arcsec in all images. However, because during the first burst the dimming stretches northward and during the third burst it does it southward, we have centered differently the top and bottom row images. The $x$ axis runs from $-100(-25)$ to 50 (125) arcsec, while the $y$ axis runs from $0(-25)$ to 135 (110) arcsec, for the top (bottom) row images.

EUV evolution of the three events. Mostly a small shortening of the emitting region is seen between 6:18 and 6:35 UT.

The EUV emission shows elongated sigmoidal loops in later images (this is more evident in the image at 8:31 UT on 11 May in Fig. 5). Then, a highly non-potential configuration was still present (and partly rebuild) before the third X-ray event (Sect. 2.3). This third event is associated to the largest changes in EUV emission (shortening of the emitting region and appearance of a cusp and extended dimmings as shown in Fig. 5, bottom).

In the EIT image at 11:08 UT (see Fig. 4), well after the third X-ray burst, the elongated loops have disappeared. The last images in Fig. 4 show how the EUV brightness slowly decreases and the bright point regains its compact structure again, before disappearing completely.

\section{Physical interpretation of the coronal eruptions}

\subsection{Observational evidences for the eruptions}

Dimmings, i.e. local decreases in emission that indcate loss and/or depletion of mass, are often observed in connection with eruptive events (Sterling \& Hudson 1997; Zarro et al. 1999; Thompson et al. 2000). Dimming regions have especially been identified after the estimated rising time of a CME, being best observed on the disc during halo events. In the studied case, EUV dimmings were clearly observed associated with the events starting after 00:42 and 08:03 UT (first and third events), while they were not observed after the second X-ray burst starting after 06:00 UT.

The top row of images in Fig. 5 illustrates the first event. At 00:35 UT the bright point is relatively intense in an extended area. Then, the X-ray burst occurs and only the central part remains bright. A compact dimming region is also observed extending towards the North-East, while no reduced emission appears to the South-West. The dimming region has a loop-like shape and does not seem to overlay much of the original EIT core brightness. No cusp is seen associated with this event.

The evolution of the dimming regions corresponding to the third X-ray burst (starting after 08:03 UT) is illustrated in the bottom row of Fig. 5. The image at 08:31 UT shows the erupting loops and an elongated bright core. By 08:48 UT, these loops have disappeared, the central part of the AR has brightened further and extended dimmings are visible both at the North-East and South-West. In the third image at 09:05 UT, the dimmings are well defined, they are more marked than in the previous case and a cusp structure has formed above the bright point.

Summarizing, the largest event is the third one in terms of integrated X-ray flux, EIT brightness disappearance around the core of the AR and EIT dimming extension. The other two events might have been associated to the eruption of large scale loops, which involved only a small fraction of the bipole 
magnetic flux. The third event implies a more global instability of the bipole's magnetic configuration. We analyze the associated dimmings in detail in the next section.

\subsection{Magnetic flux involved in dimmings}

Dimmings are classically interpreted as a transfer of plasma from the low corona into the interplanetary medium, due to the transition from closed to "open" field lines (e.g., Sterling \& Hudson 1997). However, it is worth noting that an expansion of the flux tubes, initially containing the emitting plasma, is sufficient to produce a decrease of the plasma density and, therefore, of the emissivity (that scales with the density to the second power). Gibson \& Low (2000) have shown this by calculating the X-ray emission in their analytical 3D model of an expanding magnetic flux rope. The density depletion produced the typical skewed asymmetry of the observed twin dimmings in eruptions. Then, the "opening" of the field lines to the interplanetary space is just the limit of a very large expansion, but this limit is not necessarily reached in all the dimming regions.

The magnetic flux contained in a solar ejecta is an important global quantity that can be used to link the coronal observations to the interplanetary ones. An upper limit to this quantity can be estimated considering the magnetic flux contained in the dimmings (assuming that the photospheric magnetic flux is not underestimated). As shown in Fig. 6, the dimmings cover both part of the small AR polarities and the surrounding quiet Sun regions. The magnetic field is mainly unipolar above each AR polarity, while it has strongly mixed polarities above the quiet regions; this implies that flux measurements at the AR and the quiet regions have different degrees of uncertainty.

Above each AR polarity, the largest uncertainty comes from the location of the dimming boundary since they are time dependent and mostly clear only in difference images taken with a significant time difference (a few tens of minutes). A smaller uncertainty comes from the co-alignment between EIT and MDI that we checked independently (so, outside the main bipole), using the expected association between network magnetic field and brightenings. Considering that the dimming boundaries are determined in the magnetograms with a precision of \pm 2 EIT pixels, we obtain a flux uncertainty of about $\pm 2 \times 10^{19} \mathrm{Mx}$. The magnetic flux in the dimmings above the AR bipole are almost balanced: $F_{\mathrm{AR}, \text { dimming }}=12 \pm 2 \times 10^{19} \mathrm{Mx}$, which is about $60 \%$ the magnetic flux present in each polarity at that time.

The amount of flux found in the dimmings in the quiet Sun regions, $\approx 15 \times 10^{19} \mathrm{Mx}$, is comparable in magnitude to the above value $F_{\mathrm{AR} \text {,dimming }}$ for both positive and negative polarities separately (see Fig. 7 for $B_{\text {threshold }}=0$ ), but positive and negative flux are almost balanced. Moreover, this flux is present in many small and nearby polarities. Part of this field is noise and part has a solar origin, being formed by small scale connections. In both cases, the associated flux is not part of the "open" flux. In order to avoid these sources of flux we compute the fluxes taking only the magnetogram pixels which are above a given $B_{\text {threshold }}$ (Fig. 7). The North (resp. South) dimming has a slightly negative (resp. positive) dominance in agreement with

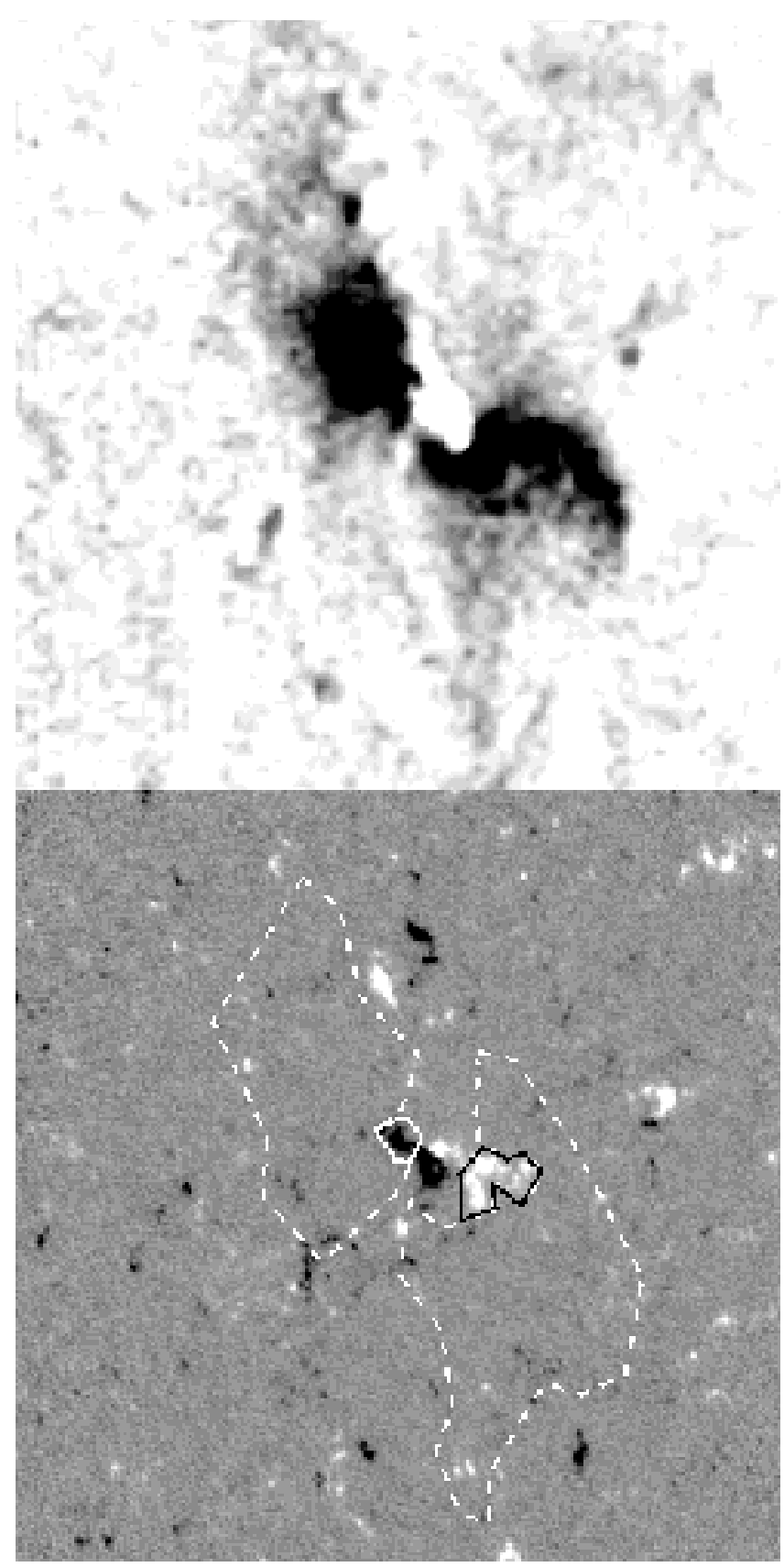

Fig. 6. Top: difference images from EIT $195 \AA$ at 08:48-08:31 UT showing the extension of the dimmings after the event starting after 08:03 UT (see Fig. 2, bottom). Bottom: MDI high resolution magnetogram closest in time with the overlaid contour of the dimmings. We have separated the dimmings North-East and South-West of the bipole and for each the extension above the small AR (continuous white/black lines on the negative/positive polarity) from that above the quiet Sun (dashed lines). Both images are $200 \times 200$ arcsec in size.

the expected dominance due to flux diffusion from the small AR nearby polarity. However, this dominance is weak for all the $B_{\text {threshold }}$ values and there is a sharp decrease in the measured flux from $B_{\text {threshold }}=0$ to $\approx 15 \mathrm{G}$, confirming that most of the flux is in weak field regions.

We conclude that the net flux in the dimmings above the quiet solar regions is small, $\approx 0.5$ up to $1.0 \times 10^{19} \mathrm{Mx}$, so less than $8 \%$ of the flux in the dimmings over the small AR. Then, 

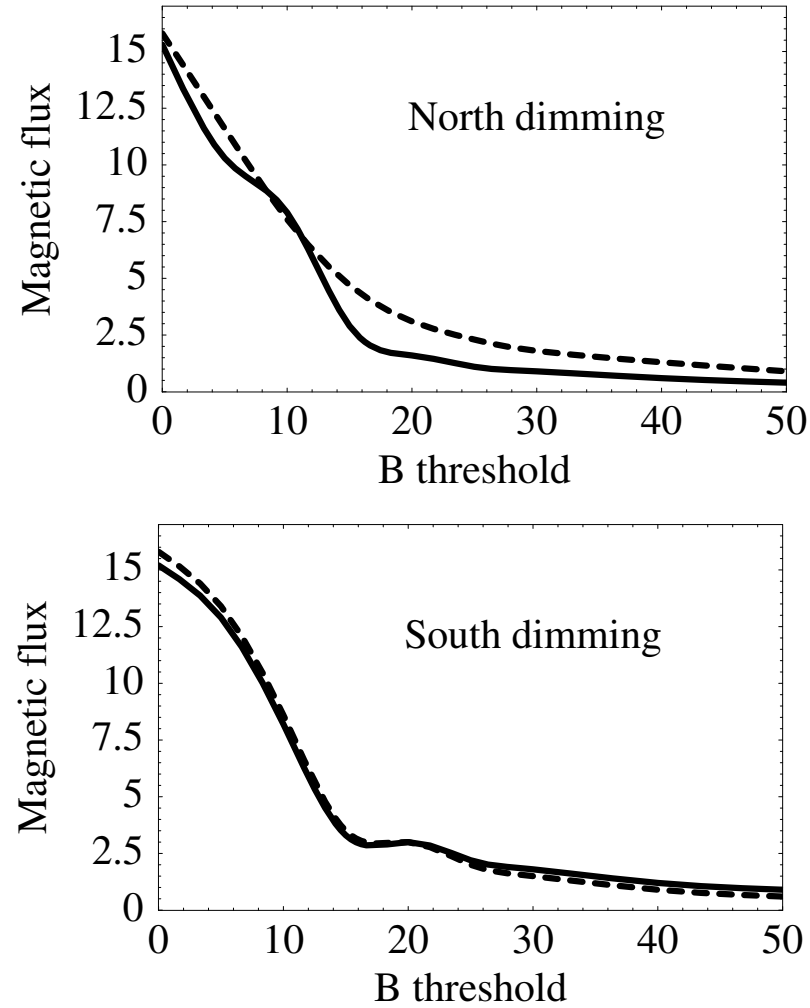

Fig. 7. Flux, in units of $10^{19} \mathrm{Mx}$, measured in the dimmings in the quiet Sun (so, excluding the portion overlying the small AR, see the dashed lines shown in Fig. 6). Positive and negative field values are summed up separately and represented with continuous and dashed lines, respectively. Low magnetic flux densities are mostly due to noise or low scale magnetic connectivities. To derive the large scale flux only, the magnetic flux densities are filtered using a minimum threshold value.

the total net flux in the dimmings (quiet Sun regions and part of AR polarities) is $F_{\text {dimming }}=13 \pm 2 \times 10^{19} \mathrm{Mx}$.

\subsection{Coronal magnetic field}

Coronal observations enable us to identify the few magnetic flux-tubes which are significantly heated, and therefore are denser and hotter than the surrounding ones. But the variable emission along the loops, the multiple loops along the line-ofsight, and the limited spatial resolution all contribute to degrade the information. We can obtain complementary information by computing the coronal magnetic field. Using MDI magnetograms, we have extrapolated the observed photospheric line of sight component of the field to the corona under the linear (or constant $\alpha$ ) force-free field assumption: $\boldsymbol{\nabla} \times \boldsymbol{B}=\alpha \boldsymbol{B}$. We have used a fast Fourier transform method as proposed by Alissandrakis (1981).

The value of $\alpha$ is chosen so as to best fit the observed coronal loops at a given time (Fig. 8). High resolution images are needed to identify independent loops. They were obtained with TRACE only in a short time interval before the first X-ray burst. The value of $\alpha$ is determined through an iterative process. First, we compute the coronal field assuming a given value for $\alpha$, then we compare the computed field lines to the observed TRACE
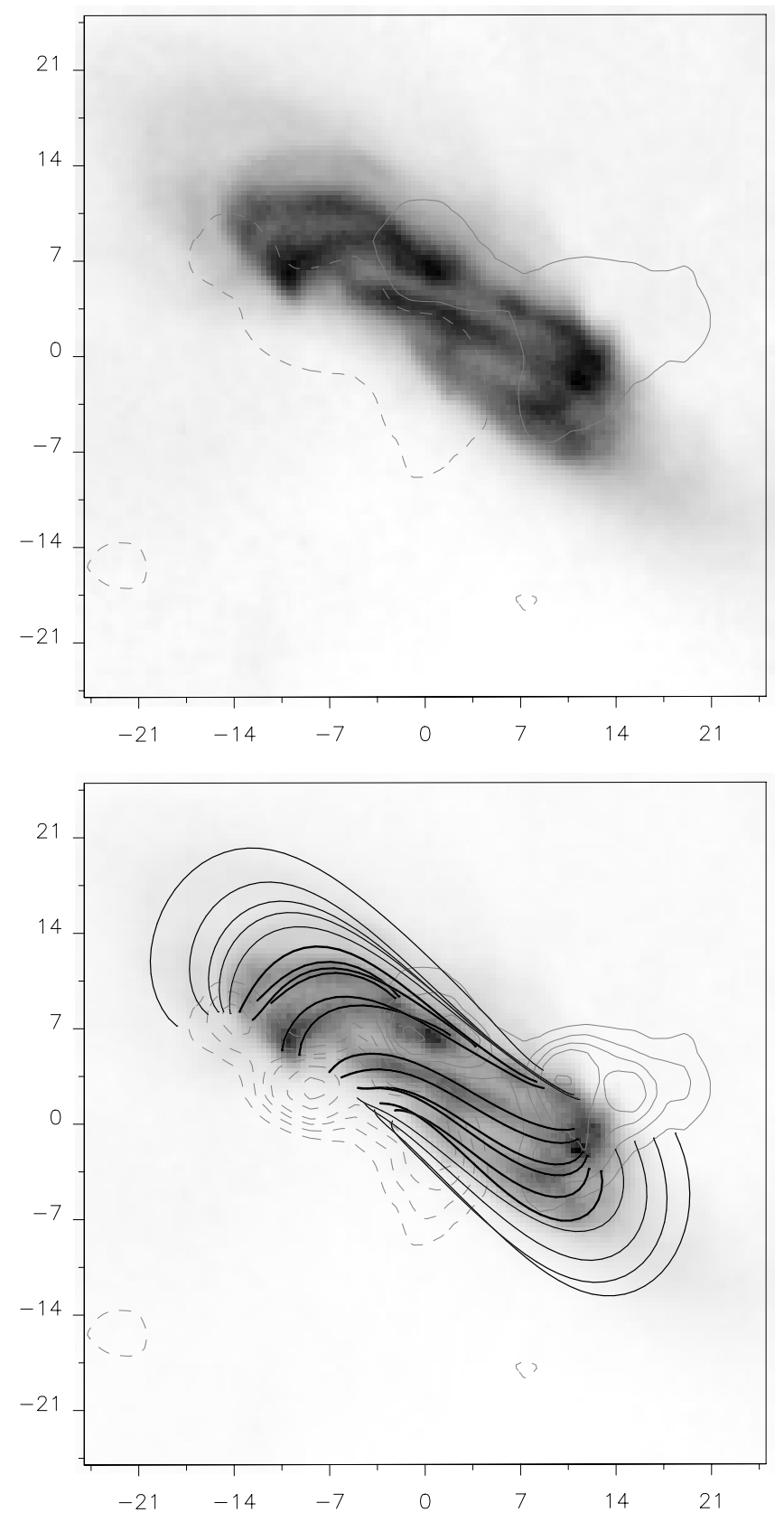

Fig. 8. Top: TRACE $195 \AA$ A observation at 00:38 UT on May 11 (reversed color) with two isocontours (grey lines) of the photospheric field taken with MDI at 00:03 UT (positive: continuous, negative: dashed). Bottom: the same image with more isocontours $( \pm 25,50$, $75,100,150,200$, grey lines) and computed field lines superimposed (the thin lines have been computed using $\alpha=-0.08 \mathrm{Mm}^{-1}$ and the thick lines using $\alpha=-0.11 \mathrm{Mm}^{-1}$ ). Notice the global $\mathrm{S}$ shape of the emission, which is a consequence of the strong non-potentiality of the magnetic field configuration. Both $\mathrm{x}$ and $\mathrm{y}$ axes are measured in $\mathrm{Mm}$.

coronal loops and, finally, through successive steps we select the value of $\alpha$ that gives the best global fit (Green et al. 2002).

As usually found before in other ARs, $\alpha$ is higher in the core of the region $\alpha=-0.11 \mathrm{Mm}^{-1}$ (thick lines in Fig. 8) than in the peripheries $\alpha=-0.08 \mathrm{Mm}^{-1}$ (thin lines). We point out that the values of $\alpha$ used to model TRACE loops are about ten times higher than any other value we have used before to 
Table 1. Coronal magnetic helicity and its variation. The values have been computed using MDI magnetograms at the times shown. This lapse of time includes the three X-ray bursts observed by SXT (see Sect. 2.3). The helicity values are in units of $10^{39} \mathrm{Mx}^{2}$.

\begin{tabular}{cccc}
\hline \hline$\alpha$ & $H_{\text {cor }}$ & $H_{\text {cor }}$ & $\Delta\left|H_{\text {cor }}\right|$ \\
$\mathrm{Mm}^{-1}$ & $00: 03 \mathrm{UT}$ & $11: 11 \mathrm{UT}$ & \\
\hline-0.08 & -5.2 & -2.9 & -2.3 \\
-0.11 & -7.5 & -4.2 & -3.3 \\
\hline
\end{tabular}

model coronal field observations, meaning that the coronal field is strongly non-potential. The central field lines are S-shaped, while the surrounding lines are J-shaped. By comparing the magnetic extrapolation with the observed loops in Fig. 8 we get a better idea of the highly sheared magnetic configuration.

\subsection{Magnetic helicity content in the corona}

An important physical quantity to link coronal observations to interplanetary ones is the magnetic helicity, because it is an almost conserved quantity. Once the coronal model is determined, we compute the relative coronal magnetic helicity, $H_{\text {cor }}$, following Berger (1985). When $\alpha$ exceeds a certain critical value $\left(\alpha_{\text {crit }}\right)$, which depends on the size of the integration box, $H_{\text {cor }}$ may take very high unphysical values (see Green et al. 2002, mainly their Appendix). A way to avoid this artificial enhancement of $H_{\text {cor }}$ when $\alpha$ lies close to $\alpha_{\text {crit }}$, which is the case for our coronal model, is to use a linearized version of the expression given by Berger (1985, see his Eq. (A23)) as discussed in Green et al. (2002, see their Eq. (11)). We note that there were typographic errors in the published equation; however, these typos appeared only in the script and did not influence the results. The correct expression is:

$H_{\text {cor }}=\alpha \sum_{n_{x}=0}^{N_{x}} \sum_{n_{y}=0}^{N_{y}} \frac{\left|\widetilde{B}_{n_{x}, n_{y}}^{2}\right|}{\left(k_{x}^{2}+k_{y}^{2}\right)^{3 / 2}}$,

where the mode $n_{x}=n_{y}=0$ (uniform field) has no contribution. Following this approach, we have obtained the results listed in Table 1.

When a flux tube is ejected from the solar corona into the interplanetary medium, it carries part of the magnetic helicity contained in the coronal field. Therefore, we need to compute the variation of the coronal magnetic helicity before and after an eruptive event to compare this coronal global quantity to the corresponding one in the associated interplanetary event. Unluckily, TRACE images are only available before the first $\mathrm{X}$-ray burst. Furthermore, due to the low intensity and spatial resolution of both SXT and even EIT, it is difficult to distinguish the shape of individual coronal loops and to use their images to determine the value of $\alpha$.

Taking a conservative approach to determine a lower bound for the variation of the coronal magnetic helicity, we select the closest in time MDI magnetogram after the third X-ray burst (starting at $\approx 8: 03 \mathrm{UT}$ ) and, using the previously determined values for $\alpha$, we computed $H_{\text {cor }}$. The results are shown in the second row of Table 1 . The minimum decrease of $\left|H_{\text {cor }}\right|$ is in the range of $-3.3 \times 10^{39} \mathrm{Mx}^{2} \leq \Delta\left|H_{\text {cor }}\right| \leq-2.3 \times 10^{39} \mathrm{Mx}^{2}$. In fact $\alpha$ is expected to decrease after the eruption which implies a larger decrease of $\left|H_{\text {cor }}\right|$. The largest possible variation is obtained if the magnetic field fully relaxes to a potential configuration after the event. The latter case gives: $-7.5 \times 10^{39} \mathrm{Mx}^{2} \leq$ $\Delta\left|H_{\text {corl }}\right| \leq-5.2 \times 10^{39} \mathrm{Mx}^{2}$. The real range for $\Delta\left|H_{\text {cor }}\right|$ should be somewhere in between the two ranges of values.

\section{Observational evidence of the interplanetary flux rope}

\subsection{In situ measurements at $1 \mathrm{AU}$}

We analyze interplanetary data around the expected time of arrival, $\approx 2-5$ days later than the coronal event, with the hope to identify the interplanetary manifestation of the main sigmoidal eruption observed at 9:00 UT on 11 May (third X-ray burst). From 10:00 UT on 13 May to 04:00 UT on 16 May, the spacecraft Wind (located in the vicinity of the Lagrangian point, L1) observed low values of the radial velocity $\left(V_{\mathrm{r}}\right)$ of the solar wind $\left(300 \mathrm{~km} \mathrm{~s}^{-1} \leq\left|V_{\mathrm{r}}\right| \leq 400 \mathrm{~km} \mathrm{~s}^{-1}\right)$. A very extended region containing plasma with a high intensity and disordered magnetic field, and low proton $\beta$ (being $\beta$ the ratio of the plasma to the magnetic pressure), was also observed by Wind between 15-17 May, 1998. The signatures just described are typical of complex interplanetary ejecta. We selected one minute resolution magnetic data along this period, and found that the duration of the complex ejecta is around $50 \mathrm{~h}$. The data were downloaded from the public site: http://cdaweb.gsfc.nasa.gov/cdaweb/istp-public/.

Despite the fact that to identify an individual flux rope within a complex ejecta at $1 \mathrm{AU}$ is highly improbable (Burlaga et al. 2002), in this particular case we are able to isolate a small event lasting from 22:00 UT on 15 May to 01:50 UT on 16 May (Fig. 9), around 4 days and $14 \mathrm{~h}(110 \mathrm{~h})$ after the sigmoidal eruption. We found this small interplanetary event by scanning the full temporal evolution of the ejecta and separating the data in different sections. Considering an average speed of $\sim 350 \pm 50 \mathrm{~km} \mathrm{~s}^{-1}$, we expect a travel time of $\sim 119 \pm 17 \mathrm{~h}$ from the corona to $1 \mathrm{AU}$; then, the small event is a good candidate to be the interplanetary manifestation of the coronal eruption since we found a very good agreement with the estimated delay. The identified interplanetary event shows a large and coherent rotation of the component $B_{y, \mathrm{GSE}}$, for about $4 \mathrm{~h}$ (beginning and end indicated by dashed vertical lines in the two upper panels of Fig. 9). This is consistent with the observation of a cylindrical flux rope as it crosses the Wind spacecraft. The coherent rotation of the magnetic field vector (Fig. 9, bottom panel), together with a high magnetic intensity and low proton temperature, indicate that this small event falls within the definition of MCs (Burlaga et al. 1981). This is one of the smallest MCs identified in in situ data till now. The velocity profile of the cloud (not shown) is almost linear with an average velocity $(v)$ $\approx 350 \mathrm{~km} \mathrm{~s}^{-1}$. It is expanding, with an expansion factor $f \sim 8 \%$ $\left(f=\left(v_{\text {end }}-v_{\text {start }}\right) /\langle v\rangle\right)$. 

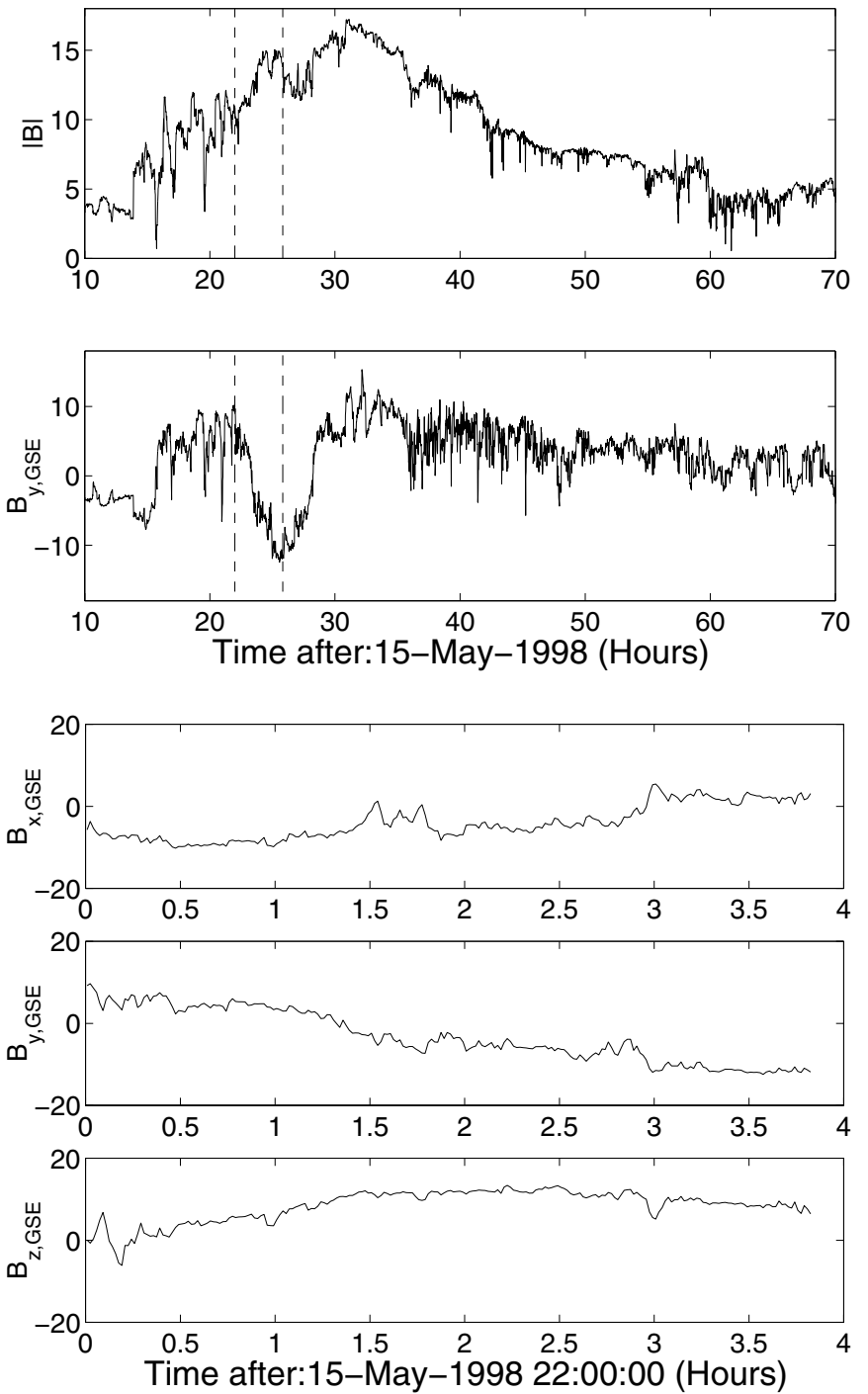

Fig. 9. Interplanetary magnetic field data obtained by the Wind spacecraft. The two upper panels show the complex ejecta (with the small MC located in between the dashed lines), and the three lower panels are a zoom on the small MC. We plot the magnetic field intensity $(|B|)$ and its components in Geocentric Solar Ecliptic (GSE) coordinates as a function of time.

\subsection{Determining the geometry of the $M C$}

When modeling an MC, we need to define a system of coordinates fixed to the cloud. Assuming that the MC has a cylindrical helical magnetic structure, the natural coordinates to describe its magnetic field are the polar ones $(r, \phi, z)$, such that $\boldsymbol{B}(r)=B_{z}(r) \hat{z}+B_{\phi}(r) \hat{\phi}$. We can define a cartesian system of coordinates fixed to the cloud, such that $\hat{z}_{\text {cloud }}$ is parallel to the axis of the cloud and $B_{z}$ is positive at this axis. $\hat{\boldsymbol{x}}_{\text {cloud }}$ is the unit vector defined by the projection of the spacecraft trajectory orthogonal to $\hat{z}_{\text {cloud }}$, and $\hat{\boldsymbol{y}}_{\text {cloud }}$ is such that the system is right-handed.

When the spacecraft's path intersects the axis of the ideal cloud defined above, the magnetic field data obtained by the spacecraft will show: $B_{x, \text { cloud }}=0$, a large and coherent variation of $B_{y, \text { cloud }}$ from positive to negative (or from negative to positive, depending on the sign of the magnetic helicity), and an intermediate and coherent variation of $B_{z, \text { cloud }}$, from low values at one cloud edge, taking the largest value at its axis and returning to low values at the other edge (Bothmer \& Schwenn 1998).

We determine the orientation of the observed magnetic cloud using a minimum variance (MV) analysis of the magnetic observations (Sonnerup \& Cahill 1967). This method finds the direction $(\hat{\boldsymbol{n}})$ in which the mean quadratic deviation of the field, $\left\langle(\boldsymbol{B} \cdot \hat{\boldsymbol{n}}-\langle\boldsymbol{B} \cdot \hat{\boldsymbol{n}}\rangle)^{2}\right\rangle$, is minimum (maximum). It is possible to show that this is equivalent to finding the eigen-vector corresponding to the smallest (highest) eigenvalue of the covariance matrix $M_{i, j}=\left\langle B_{i} B_{j}\right\rangle-\left\langle B_{i}\right\rangle\left\langle B_{j}\right\rangle$. As we mentioned above, when the minimum distance from the spacecraft to the axis of the flux tube is close to zero, the largest (resp. smallest) change of $\boldsymbol{B}$ comes from the component of the field associated with $B_{y}$ (resp. $B_{x}$ ). Thus, this MV method determines the direction of the maximum $\left(\hat{\boldsymbol{y}}_{\text {cloud }}\right)$, intermediate $\left(\hat{z}_{\text {cloud }}\right)$, and minimum $\left(\hat{\boldsymbol{x}}_{\text {cloud }}\right)$ variance of the field. A more complete discussion of the MV method applied to interplanetary flux tubes can be found in the appendix of Bothmer \& Schwenn (1998).

In our event, we find a well-defined direction for the axis of the MC (corresponding to the intermediate eigen-vector) since we get an intermediate to minimum eigenvalues ratio of $\sim 6$. We define the MC axis orientation as follows: the angle $\theta$ between the ecliptic plane and the MC axis, and the angle $\varphi$ between $\hat{\boldsymbol{x}}_{\mathrm{GSE}}$ and the projection of the MC axis on the ecliptic plane (measured positive when counterclockwise). We find that $\theta \sim 59^{\circ}$ and $\varphi \sim 172^{\circ}$, so the projection of the axis of the cloud on the ecliptic plane lies almost along $\hat{\boldsymbol{x}}_{\mathrm{GSE}}$; in particular, with the magnetic field pointing towards $-\hat{\boldsymbol{x}}_{\mathrm{GSE}}$.

In this approach, the spacecraft impact parameter (the minimum distance from the spacecraft trajectory to the axis of the cloud), $p$, is not determined and we set it equal to zero, noting that the large angle rotation of the field $\left(\sim 147^{\circ}\right.$, see left upper panel of Fig. 10) indicates that the ratio between the impact parameter and the radius of the cloud $(p / R)$ should be small. This field rotation also implies that the sign of the helicity is negative. Next, from the measured velocity, the spacecraft trajectory through the cloud and the time along which Wind observed the cloud, we estimate a radius $R \sim 1.6 \times 10^{-2} \mathrm{AU}$, which implies a very small MC.

\subsection{Modelling the MC magnetic field}

In situ measurements of interplanetary magnetic flux tubes at $1 \mathrm{AU}$ are consistent with several axially symmetric magnetic field models. The most frequently used is the linear force-free model of Lundquist (1950), $\boldsymbol{\nabla} \times \boldsymbol{B}=\alpha \boldsymbol{B}$, with $\alpha$ constant (e.g., Burlaga et al. 1981; Burlaga 1988; Lepping et al. 1990). For helical-cylindrical configurations of the field, the amount by which a given field line is twisted per unit length, i.e., the twist $\tau(r)=\mathrm{d} \phi / \mathrm{d} z=B_{\phi} /\left(r B_{z}\right)$, depends only on $r$. In particular for the Lundquist's model, the twist when $r \rightarrow 0$ is $\tau_{0}=\alpha / 2$. A cylindrical non-linear force-free field with a uniform twist, i.e. $\tau(r)=\tau_{0}$ (Gold \& Hoyle 1960), is also used to model interplanetary flux tubes (e.g., Farrugia et al. 1999). The cylindrical non force-free model proposed by Hidalgo et al. $(2000,2002)$ 

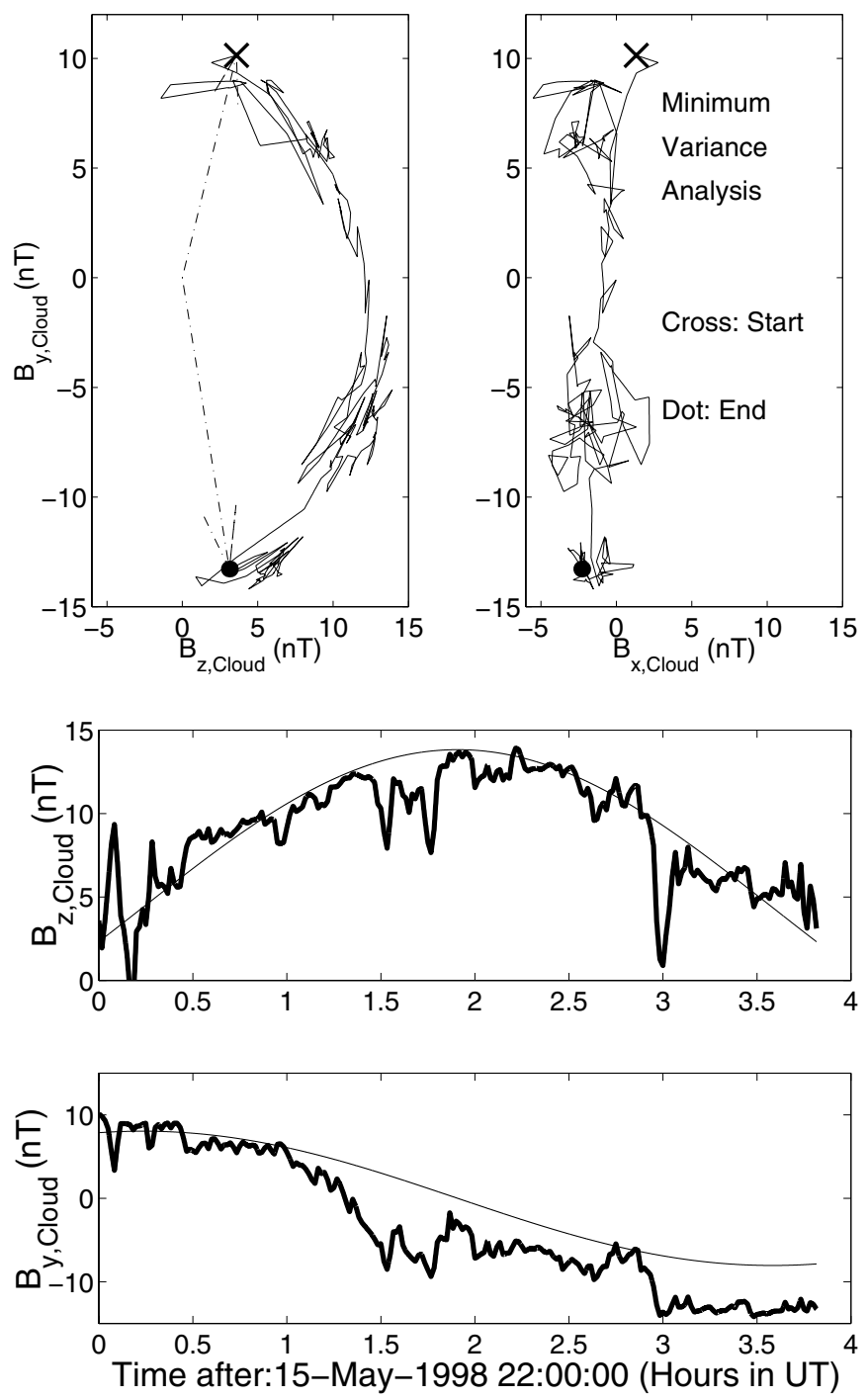

Fig. 10. Magnetic field components in local MC coordinates. The two upper panels show the hodograms for the MC. The central and lower panels show the evolution of the magnetic field components (with the orientation of the cloud given by the minimum variance method, see Sect. 4.2). The observed magnetic data are drawn with thick lines, and the best solutions fitted using Lundquist's model are shown with thin lines.

assumes a constant current density such that $\boldsymbol{j}(\boldsymbol{r})=j_{\phi} \hat{\phi}+j_{z} \hat{z}$, where $j_{\phi}$ and $j_{z}$ are constants.

The magnetic field of these three different cylindrical flux tube configurations can be obtained as a function of $r$ (the radius), $B_{0}$ (the field at the flux tube axis), and $\tau_{0}$ (the twist at the flux tube axis), for detailed expressions see Dasso et al. (2003b).

We use the orientation found from the MV method to model the observed field, according to the three models described above, and we obtain the two physical parameters $\left(\tau_{0}, B_{0}\right)$ that best fit the observations following the method discussed in Dasso et al. (2003a,b). The two lower panels in Fig. 10 correspond to $B_{z, \text { cloud }}$ and $B_{y, \text { cloud }}$ for the small MC. The observed data are shown with thick lines and the curves obtained using the parameters fitted to the Lundquist's model are shown with thin lines. The other two models (Gold-Hoyle \& Hidalgo et al.) give similar curves.

Table 2 shows the fitted values for $\tau_{0}$ and $B_{0}$ for the three models. The value of $B_{0}$ is very similar in the three models ( 14-16 nT). The twist of the field lines at the center of the tube is lower for Hidalgo's et al. model and larger ( $\sim 70 \%$ with respect to its lowest value) for Gold-Hoyle's model.

\subsection{Global characteristics of the MC}

Magnetic flux and magnetic helicity $\left(H_{\text {cloud }}\right)$ are two important global physical quantities that characterize the magnetic field configuration in interplanetary clouds. They are also useful to link interplanetary events to their coronal source region.

Using the three models described in the previous section, we can find analytical expressions for the flux $\left(F_{z}\right)$ of $B_{z}$ (i.e., along the flux tube) and also for the flux $\left(F_{\phi} / L=\int_{0}^{R} \mathrm{~d} r B_{\phi}(r)\right)$ of $B_{\phi}$ per unit length along the MC. The value of $F_{z}$ can be computed directly from the distribution of $B_{z}$ for every model. Concerning $F_{\phi} / L$, for the Lundquist's model, we have: $F_{\phi}^{L} / L=B_{0}\left[1-J_{0}(\alpha R)\right] / \alpha$, where $J_{0}$ is the Bessel function of the first kind and zero order. For the Gold-Hoyle model, we find: $F_{\phi}^{\mathrm{GH}} / L=B_{0} \ln \left(1+\tau_{0}^{2} R^{2}\right) /\left(2 \tau_{0}\right)$. While, for the Hidalgo's et al. model, we have: $F_{\phi}^{\mathrm{H}} / L=\tau_{0} B_{0} R^{2} / 2$.

In a cylindrical magnetic field configuration, a simple expression for the relative helicity content per unit length in the cloud is given by: $H_{\text {cloud }} / L=4 \pi \int_{0}^{R} A_{\phi}(r) B_{\phi}(r) r \mathrm{~d} r$, where $A_{\phi}$ is the component $\hat{\phi}$ of the potential vector. Thus, for the Lundquist's model, the helicity writes: $H_{\text {cloud }}^{L} / L=$ $8 \pi U^{-4}\left(\int_{0}^{U} J_{1}^{2}(u) u \mathrm{~d} u\right) B_{0}^{2} R^{4} \tau_{0}$, where $J_{1}$ is the Bessel function of the first kind and first order. The variables $u=2 \tau_{0} r$ and $U=2 \tau_{0} R$ are dimensionless quantities. For the GoldHoyle model, the helicity is given by: $H_{\text {cloud }}^{\mathrm{G}} / L=(8 \pi[\ln (1+$ $\left.\left.\left.U^{2} / 4\right)\right]^{2} U^{-4}\right) B_{0}^{2} R^{4} \tau_{0}$. Finally, for the Hidalgo's et al. model we have: $H_{\text {cloud }}^{\mathrm{H}} / L=(7 \pi / 30) B_{0}^{2} R^{4} \tau_{0}$.

The magnetic flux of $B_{z}$ has a narrow range of values in the force-free cases (seventh column in Table 2), $F_{z} \sim 1.3-1.4 \times$ $10^{19} \mathrm{Mx}$, but it changes by $\sim-30 \%$ for Hidalgo's et al. model. $F_{z}$ for this small MC is about one hundredth of the average value for large $\mathrm{MCs}\left(F_{z} \sim 10^{21} \mathrm{Mx}\right)$. The same behaviour is found for the magnetic flux of $B_{\phi}$ per unit length for the two force-free models, $F_{\phi} / L \sim 19 .-20 . \times 10^{19} \mathrm{Mx} / \mathrm{AU}$; while it changes only by $\sim 15 \%$ for Hidalgo's et al. model.

The magnetic field of the flux tube is left-handed so $H_{\text {cloud }}$ is negative, and it is in the range $\left|H_{\text {cloud }}\right| / L \sim(2.8-3) \times$ $10^{39} \mathrm{Mx}^{2} / \mathrm{AU}$ for the three models (last column in Table 2). Note that the two force-free models give practically the same magnetic helicity, while the non-force free model gives a lower value for $\left|H_{\text {cloud }}\right| / L$ by $\sim 7 \%$. The magnetic helicity per unit length for this event is smaller than for typical MCs by three orders of magnitude, being the mean value of the helicity in clouds $\left|H_{\text {cloud }}\right| / L \sim 4 \times 10^{42} \mathrm{Mx}^{2} / \mathrm{AU}$ (van-Driel Gesztelyi et al. 2003). 
Table 2. Geometrical (obtained from the minimum variance method) and physical parameters (fitted with a least-square method) for the small MC. L, G, and $\mathrm{H}$ refer to Lundquist, Gold and Hoyle, and Hidalgo et al. model, respectively. The geometrical parameters are: the angle $\theta$ between the axis of the tube and the ecliptic plane, the angle $\varphi$ between the projection of the axis on the ecliptic plane and the abscissa of GSE (anti-clockwise as seen from the positive $z_{\mathrm{GSE}}$ axis), and the flux tube radius $(R)$. The two physical parameters are: the twist per unit length $\left(\tau_{0}\right)$ and the intensity of the field $\left(B_{0}\right)$, both at the flux tube centre. In the last three columns, we give the computed magnetic flux in the $B_{z}$ component $\left(F_{z}\right)$, in the $B_{\phi}$ component per unit length $\left(F_{\phi} / L\right)$ and the magnetic helicity per unit length $\left(H_{\text {cloud }} / L\right)$.

\begin{tabular}{lcccccccc}
\hline \hline Model & $\theta$ & $\varphi$ & $R$ & $\tau_{0}$ & $B_{0}$ & $F_{z}$ & $F_{\phi} / L$ & $H_{\text {cloud }} / L$ \\
& $\circ$ & $\circ$ & $10^{-2} \mathrm{AU}$ & $\mathrm{AU}^{-1}$ & $\mathrm{nT}$ & $10^{19} \mathrm{Mx}$ & $10^{19} \mathrm{Mx} / \mathrm{AU}$ & $10^{39} \mathrm{Mx}^{2} / \mathrm{AU}$ \\
\hline $\mathrm{L}$ & 59 & 172 & 1.6 & -66 & 13.8 & 1.3 & 20. & -3.0 \\
$\mathrm{G}$ & 59 & 172 & 1.6 & -85 & 14.1 & 1.4 & 19. & -2.8 \\
$\mathrm{H}$ & 59 & 172 & 1.6 & -51 & 15.9 & 0.9 & 23. & -3.0 \\
\hline
\end{tabular}

\section{Linking the coronal eruption to the interplanetary MC}

\subsection{Evidences for the link}

In Sect. 3 we have shown that the small AR at disc centre on 11 May 1998 produced three main X-ray bursts. The third one was the most significant in X-ray total flux, in EUV evolution and also in evidences for eruption. This coronal eruption and the interplanetary MC, described in Sect. 4, have properties that indicate their probable association:

- Location: eruption very close to the disc centre; as ejections travel dominantly in the radial direction it implies that the event could be observed by Wind.

- Timing: four and half days travel time what is expected for a slow CME travelling at about the speed of the slow solar wind (e.g., Vršnak \& Gopalswamy 2002) as measured in situ by Wind.

- The orientation of the MC axis is approximately the same as the direction of the elongated coronal sigmoid. Furthermore, the signs of the axial magnetic field in the $\mathrm{MC}$ and of the AR polarities are the same.

- The sign of the magnetic helicities of the MC and the small $\mathrm{AR}$ are in agreement.

Any of the above correspondences between the physical characteristics on the Sun and in the interplanetary medium could happen by chance. However, the probability that all these characteristics coincide simultaneously just by chance is small. We conclude that the observed small MC is most probably the consequence of the observed coronal eruption. This is summarized in Fig. 11. Furthermore, from the interplanetary magnetic field (Fig. 9), the orientation of the MC and the geometry of the coronal magnetic field (Fig. 8), we can deduce that the Wind spacecraft crossed the positive (western) leg of the flux rope. This is coherent with the position of the small active region at the time of the eruption ( 2 degrees of longitude East on the Sun) if the ejection occurred mostly in the radial direction.

\subsection{Critical discussion of the evidences}

Several ARs were present on the Sun at the time of our $\mathrm{X}$-ray bursts and a number of flares in the high-B to low-C GOES range occurred from May 11 to May 12 (see http:// wWw. sec.noaa.gov/weekly/pdf1998/prf1185.pdf). The third X-ray burst discussed here could be identified as the B6.9 GOES event reported by NOAA that was not associated to any of the numbered ARs on the disc and reached maximum at 09:03 UT.

Within the next $34 \mathrm{~h}$ there were 7 more flare events, 5 of them slightly more intense, while the other two of similar magnitude as the third X-ray burst. Of these events, the B8.4 flare starting on 11 May at 12:54 UT and the C1.2 flare starting on 12 May at 05:07 UT can be regarded as long-duration events, which are usually thought to be eruptive (see some examples in van Driel-Gesztelyi et al. 2000). However, these two events (as most of the events during 11 and 12 May) occurred in AR 8218 , which was located at S20E15 on 11 May at $\approx 08: 00$ UT (see Fig. 1). The loops in AR 8218, as those in another decaying not numbered AR located at $\approx \mathrm{S} 25 \mathrm{~W} 20$, had a global forward S-shape which is considered as an indication of positive twist (see Pevtsov \& Balasubramaniam 2003, and references therein). Since the SXT image in Fig. 1 has been enhanced in order to better show the small bright point, saturating brighter loops, we refer the reader to http://surfwww.mssl.ucl. ac.uk/surf/sxt/images/sxt_980511.120226.gif for a better view of the loops in AR 8218. This positive twist in southern hemisphere ARs is what is expected according to the hemispheric chirality rules (Pevtsov \& Balasubramaniam 2003). Therefore, none of the events occurring in AR 8218 could have given origin to the small MC observed by Wind, which had a negative magnetic helicity.

Concerning the orientation of the elongated coronal sigmoid, if the flux tube goes through a kink instability during the eruption (as suggested by some numerical simulations, see e.g. Fan \& Gibson 2003; Török \& Kliem 2004) part of the negative twist would be traded for a negative writhe; this will turn the flux tube axis clockwise. However, we have no evidence that the ejected flux tube was kinked and, furthermore, numerical simulations do not follow the evolution in the interplanetary space. Moreover, we concluded that the spacecraft crossed the western leg of the flux rope (i.e., the calculated MC orientation corresponds to the orientation of that portion of the flux tube); so, the orientation of the leading part of the flux rope is not known.

\subsection{More evidences: Magnetic flux and helicity}

Next, we attempt to quantify this link with the measured magnetic fluxes and helicities. For the comparison of the coronal 


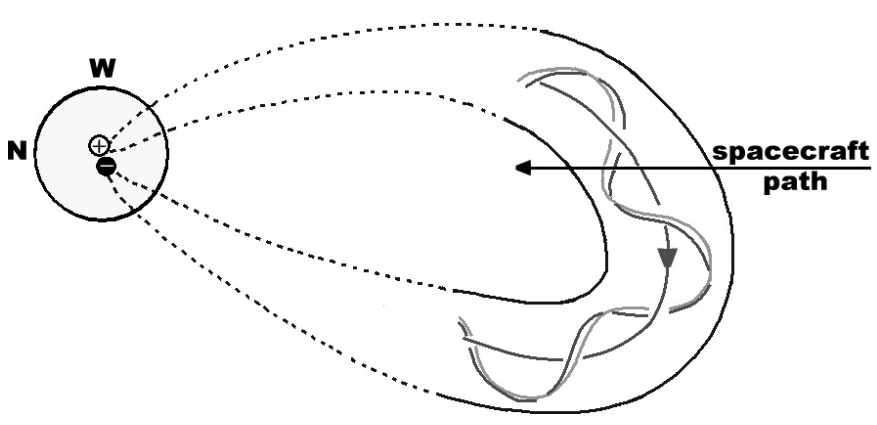

Fig. 11. Schematic global view of the magnetic cloud and its source region (notice that in this figure the solar North is to the left). The MC leading part is represented by continuous lines, while dashed lines are used closer to the Sun since, considering the photospheric magnetic observations, the MC should be detached from its solar source by the time it was observed by the Wind spacecraft.

and interplanetary magnetic helicities, the main unknown is the distribution of the twist along the interplanetary flux rope. Even supposing a uniform distribution, the MC length is unknown. In large-scale MCs, there is frequently evidence of their magnetic connection to the Sun when they are observed at $1 \mathrm{AU}$ (e.g. presence of bi-streaming electrons). This leads to an MC length slightly larger than $2 \mathrm{AU}$. However, for the studied MC, we know that the photospheric magnetic bipole disappeared about one day after the eruption (Fig. 2), so the erupting flux rope was well detached from its original solar source when it was observed in situ $\approx 4.5$ days after the coronal eruption. A simple proportionality gives a length of $\approx 0.5 \mathrm{AU}$. However, after reconnection with large-scale field lines, the magnetic twist contained in the ejected flux tube propagates along the new connections as a torsional Alfvén wave. Taking a typical Alfvén velocity of $100 \mathrm{~km} \mathrm{~s}^{-1}$, the twist can propagate on both sides of the flux tube over a length of $0.2 \mathrm{AU}$ in 3.5 days. We conclude that the probable length of the observed MC is between 0.5 and 1 AU.

We found that the flux in the dimmings is $F_{\text {dimming }}=$ $13 \pm 2 \times 10^{19} \mathrm{Mx}$. To which of the two flux values computed for the MC should this value be compared will depend on the way the ejection occurs. If the MC would result from a simple expansion of coronal loops, then $F_{\text {dimming }}$ would correspond to the flux of the axial field component of the MC $\left(B_{z}\right)$. However, we have found that $F_{z}$ is only one tenth of $F_{\text {dimming }}$. If the ejection is the result of the expulsion of a twisted flux tube formed during the eruption by successive reconnections in a sheared magnetic arcade (as suggested by Démoulin et al. 1996; Titov \& Démoulin 1999, see a sketch in Fig. 12), which we consider as the plausible scenario in our case (see the discussion in Sect. 2.5); then, $F_{\text {dimming }}$ should be closer to the value of the flux in the azimuthal component of the cloud $\left(B_{\phi}\right)$. The value of $F_{\phi} / L$ is in the range 19 to $23 \times 10^{19} \mathrm{Mx} \mathrm{AU}^{-1}$. Considering a length between 0.5 and $1 \mathrm{AU}$ for the cloud, we find: $9.5 \times 10^{19} \mathrm{Mx} \leq F_{\phi} \leq 23 \times 10^{19} \mathrm{Mx}$, so $F_{\phi}$ is in good agreement with $F_{\text {dimming }}$.

The estimated cloud helicity is $\left|H_{\text {cloud }}\right| \approx 1.5$ to $3 \times$ $10^{39} \mathrm{Mx}^{2}$, so in the range of $0.5-1.3$ the variation of helicity found at the coronal level $\left(\Delta\left|H_{\text {cor }}\right|\right.$ in Table 1$)$. These values are also in good agreement.
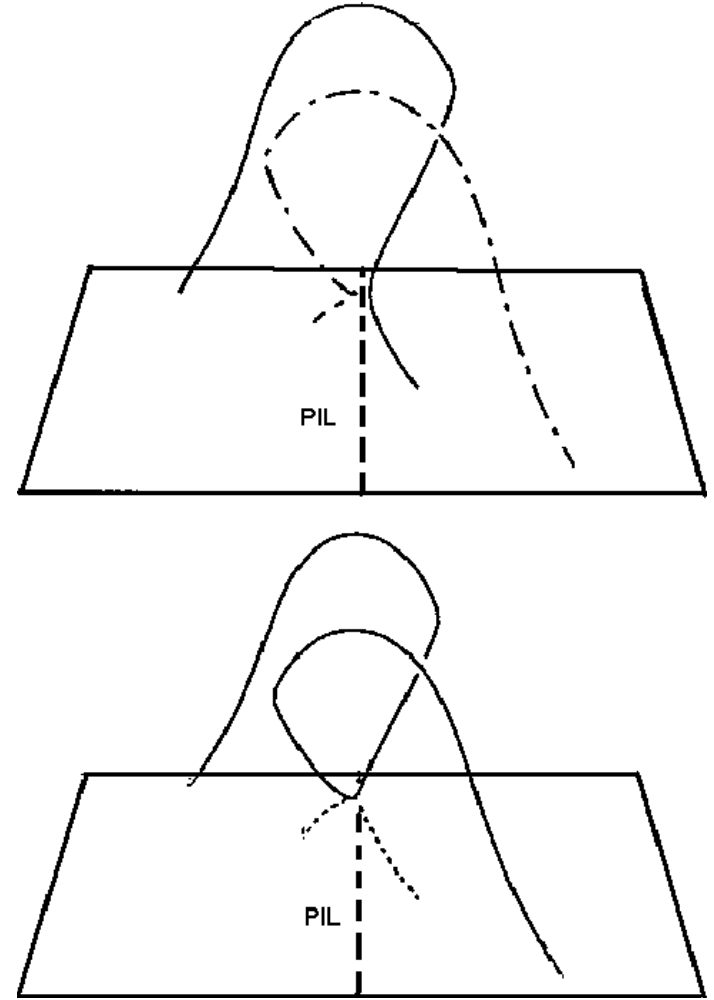

Fig. 12. A sketch showing the way a magnetic flux tube can be formed by magnetic reconnection in an expanding sheared arcade (Démoulin et al. 1996). The upper and lower figures show two field lines belonging to the expanding arcade (top), and a small arcade and flux tube (bottom), before and after reconnection respectively. PIL indicates the photospheric inversion line. Further reconnection between the flux tube and the large scale arcade will increase the number of turns in the flux tube. Coronal dimmings are expected to form in all the expanding arcade. However, only a fraction of the photospheric flux of the dimmings contributes to the longitudinal flux of the twisted flux tube; the remaining part is transferred (through reconnection) to the magnetic flux in the azimuthal component.

From the common properties between the magnetic configuration in the corona and the interplanetary space (listed in the first paragraph of this section), but also evidenced by the same order of magnetic fluxes and helicities, we conclude that the observed coronal eruption (third X-ray burst in Fig. 2) was indeed observed in the interplanetary space as a small MC. Such link could be found for such a tiny event mostly because the small AR was well isolated at disc centre, but also because a large variety of complementary solar observations, with a good time coverage, were available.

\section{Conclusions}

The studied small AR, observed from 10 to 12 May 1998 by SOHO, TRACE and Yohkoh, was especially interesting for the following reasons. First, its full evolution, from birth to full disappearance, could be observed close to the disc centre. Second, it contrasts with previous studies by its lifetime and the amount of its magnetic flux since both are a factor one hundred lower than in earlier full-evolution studies of ARs (Démoulin et al. 2002; Green et al. 2002). Third, it is a simple and isolated 


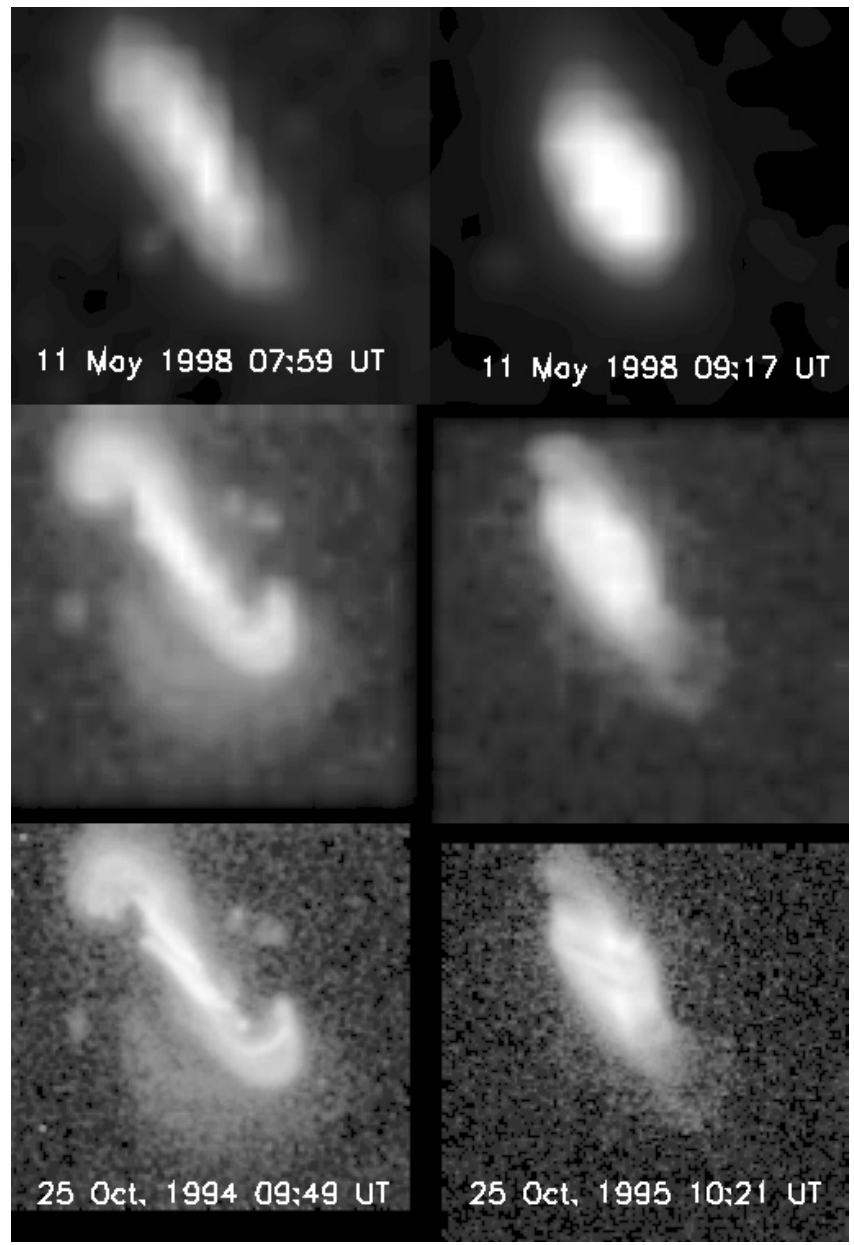

Fig. 13. Comparison to the event studied by Manoharan et al. (1996). The left (resp. right) column correspond to X-ray images at the beginning (resp. after) the eruption. The top row shows the studied X-ray bright point, as observed in SXT full disc images (the field of view is about $80 \times 80$ arcsec). The middle and bottom rows are the SXT partial frame images of the event studied by Manoharan et al. (the field of view is about $310 \times 310$ arcsec). The original images are shown in the bottom row, while their spatial resolution has been degraded (rebinned) by a factor 8 in the middle row (to account both for the difference in the observed resolution, a factor 2 , and spatial extension of the ARs, about a factor 4).

magnetic bipole, well separated from other ARs (by at least half solar radius) but it was still eruptive. Finally, an associated magnetic cloud (MC) could be identified in the interplanetary medium; this MC is also a factor one hundred smaller (in magnetic flux) than most MCs analyzed so far.

The history of this small AR can be briefly summarized as follows. The evolution of the photospheric magnetic field is consistent with the emergence of a strongly twisted $\Omega$ loop. The coronal EUV and X-ray emissions show elongated S-shaped structures situated along the magnetic inversion line of the photospheric bipole; this characterizes a strongly non-potential coronal magnetic field. Three major X-ray bursts occurred during the AR's lifetime. All of them are associated with a shortening of the coronal loops, which characterizes a relaxation to a lower magnetic energy state. Two of these bursts are associated with observed dimmings in EUV. Only the third burst is associated with the formation of a cusp in the EUV emission. This third burst also shows the most important change of shape in the EUV and the most extended dimmings (with two extended lobes); therefore, we conclude that this event was the largest eruption of this small AR.

The above summary indicates that the third event has all the observational signatures of classical eruptive events. We compare its X-ray evolution to a large-scale case (extending to one solar radius) analyzed by Manoharan et al. (1996). In both cases the magnetic bipole was mostly East-West with an inversion line slightly inclined in the North-South direction, and both cases have negative magnetic helicity (inverse-S shaped coronal emission). The high-resolution observations (SXT partial frame) of the 25 October 1994 event let us identify two sets of bright loops: long, sigmoidal ones and short, sheared ones (Fig. 13, bottom left). Mainly the short ones, analogous to classical flare loops, remain later on in the event (Fig. 13, bottom right). The sigmoidal loops progressively decrease in brightness as they expand (implying a decrease of plasma density and, then, of emission). There has most probably been an ejection towards the interplanetary space, but the associated CME could not be identified because observations were not available. These high-resolution observations let us characterize the reconnection process among the sheared loops of the bipole: as the ejection took place, the reconnection enlarged (or at least formed) a twisted flux tube; the new reconnected loops can be identified as the sigmoidal and short loops (see e.g. Démoulin et al. 1996). The presence of two sets of loops is much less obvious if we simply degrade the original images to meet the observing conditions of the much smaller 11 May 1998 event (Fig. 13, middle row). Here, we mainly observe the transition from an elongated X-ray emission (with a brighter core) to a compact emission. This is the main characteristic of the 11 May event (Fig. 13, top row). We conclude that we have basically the same phenomenon, but rescaled. The main difference between the two top rows of Figure 13, the contrast of the sigmoidal loops, is likely to be a direct effect of the scaling: in the 11 May case there is much less energy release involved; so, cooler but also much less dense coronal loops were formed, both effects contributing to a lower emission in the SXT detection range.

In the interplanetary medium, we plausibly find the associated ejecta because it shares the characteristics expected for an eruption from the small AR (Sect. 5). This small MC is also a small-scale version of previously observed classical MCs (even if it is embedded in a much larger event). This broadens our view of solar eruptions: they could also be of small scale. Indeed, taking into account the frequency of small scale bipoles on the Sun, we expect that such small ejected flux tubes would be relatively frequent in the interplanetary space (while present studies are biased by focusing on large-scale events, which are easier to identify). The relative global importance of these small-scale events in terms of magnetic flux, energy and helicity, remains to be investigated.

Such small events present a challenge for CME models, which have been developed for large scale magnetic configurations. However, since the event is very small and not fully covered by high spatial and temporal resolution data, a confrontation with any current CME model is difficult and, then, 
non-conclusive. Such confrontation would need a 3D MHD simulation of the event. Still, the analyzed event brings two constraints. First, the observations show that the magnetic stress is brought with the magnetic field by emergence and not by shearing motions due to differential rotation, which inputs a negligible amount of helicity on a time scale of one day. This confirms previous results obtained on classical ARs (Chae et al. 2001; Démoulin et al. 2002; Green et al. 2002; Nindos \& Zhang 2002). Second, the comparison of the magnetic fluxes indicates that an important fraction of the ejected flux rope is formed by reconnection of sheared arcade-like field lines.

Finally, to understand better this kind of small events, we certainly need coronal observations with both a better sensitivity (dynamic range) and spatial resolution. The next missions, Solar B and Solar Orbiter, are well suited to continue this research. Moreover, observing the associated CME is still a challenge. The configuration of the two spacecrafts of STEREO would allow us to observe such event also from a lateral point of view, from which it is easier to detect the scattered light of such a small halo CME.

Acknowledgements. The authors thank the referee, B. Kliem, for very constructive comments on the paper. The authors thank the SOHO/MDI and EIT consortia for their data, the Mullard Space Science Laboratory (MSSL) Solar UK Research Facility (SURF) for YOHKOH/SXT, and the NASA's Space Physics Data Facility (SPDF) for the interplanetary observations. The Nobeyama Radioheliograph is operated by Nobeyama Radio Observatory/NAOJ. SOHO is a joint project by ESA and NASA. S.P. acknowledges a joint travel grant from CIMO (Finland) and EGIDE (France). P.D. and C.H.M. acknowledge financial support from ECOS (France) and SECyT (Argentina) through their cooperative science program (A01U04). C.H.M. and L.v.D.G. thank TET (Hungary) and SECyT for financial support through their cooperation program (AR03/02 and HU/A01/UIII/01). P.D. and L.v.D.G. acknowledge travel support from the Royal Society Joint project (MSSL/UCL-Observatoire de ParisMeudon). C.H.M. is grateful for an MSSL/UCL visitor's grant. S.D. thanks the Observatoire de Paris, Meudon, for support during his visit. L.v.D.G. was supported by the Hungarian Government grant OTKA T-038013. C.H.M. and S.D. thank the Argentinean grants: UBACyT X329, PIP 2693 and PIP 2388 (CONICET), and PICT 12187 and PICT 14163 (ANPCyT).

\section{References}

Alissandrakis, C. E. 1981, A\&A, 100, 197

Antiochos, S. K., Devore, C. R., \& Klimchuk, J. A. 1999, ApJ, 510, 485

Berger, M. A. 1985, ApJS, 59, 433

Berger, T. E., \& Lites, B. W. 2003, Sol. Phys., 213, 213

Bothmer, V., \& Schwenn, R. 1994, Space Sci. Rev., 70, 215

Bothmer, V., \& Schwenn, R. 1998, Ann. Geophys., 16, 1

Burlaga, L., Sittler, E., Mariani, F., \& Schwenn, R. 1981, J. Geophys. Res., 86, 6673

Burlaga, L. F. 1988, J. Geophys. Res., 93, 7217

Burlaga, L. F., Plunkett, S. P., \& St. Cyr, O. C. 2002, J. Geophys. Res. (Space Physics), 107, 1266

Canfield, R. C., Hudson, H. S., \& McKenzie, D. E. 1999, Geophys. Res. Lett., 26, 627
Chae, J., Wang, H., Qiu, J., et al. 2001, ApJ, 560, 476

Démoulin, P., Priest, E. R., \& Lonie, D. P. 1996, J. Geophys. Res., 101,7631

Démoulin, P., Mandrini, C. H., van Driel-Gesztelyi, L., et al. 2002, A\&A, 382, 650

Dasso, S., Mandrini, C. H., \& Démoulin, P. 2003a, in Solar Wind Ten, AIP Conf. Proc., 546, 786

Dasso, S., Mandrini, C. H., Démoulin, P., \& Farrugia, C. J. 2003b, J. Geophys. Res. (Space Physics), 108, 1362

Delaboudiniere, J.-P., Artzner, G. E., Brunaud, J., et al. 1995, Sol. Phys., 162, 291

Dobrzycka, D., Raymond, J. C., Biesecker, D. A., Li, J., \& Ciaravella, A. 2003, ApJ, 588, 586

Fan, Y., \& Gibson, S. E. 2003, ApJ, 589, L105

Farrugia, C. J., Janoo, L. A., Torbert, R. B., et al. 1999, in Solar Wind Nine, AIP Conf. Proc., 471, 745

Fisher, G. H., Longcope, D. W., Metcalf, T. R., \& Pevtsov, A. A. 1998, ApJ, 508, 885

Gibson, S. E., \& Low, B. C. 1998, ApJ, 493, 460

Gibson, S. E., \& Low, B. C. 2000, J. Geophys. Res., 105, 18187

Gibson, S. E., Fan, Y., Mandrini, C. H., Fisher, G., \& Démoulin, P. 2004, ApJ, 617, 600

Gilbert, H. R., Serex, E. C., Holzer, T. E., MacQueen, R. M., \& McIntosh, P. S. 2001, ApJ, 550, 1093

Glover, A., Ranns, N. D. R., Harra, L. K., \& Culhane, J. L. 2000, Geophys. Res. Lett., 27, 2161

Gold, T., \& Hoyle, F. 1960, MNRAS, 120, 89

Golub, L. 1980, Royal Society of London Philosophical Transactions Series A, 297, 595

Green, L. M., López Fuentes, M. C., Mandrini, C. H., et al. 2002, Sol. Phys., 208, 43

Handy, B. N., Acton, L. W., Kankelborg, C. C., et al. 1999, Sol. Phys., 187,229

Harrison, R. A., Bryans, P., \& Bingham, R. 2001, A\&A, 379, 324

Harvey, K. L. 1993, Ph.D. Thesis

Harvey, K. L. 1997, in Magnetic Reconnection in the Solar Atmosphere, ASP Conf. Ser., 111, 9

Hidalgo, M. A., Cid, C., Medina, J., \& Viñas, A. F. 2000, Sol. Phys., 194,165

Hidalgo, M. A., Cid, C., Medina, J., Viñas, A. F., \& Sequeiros, J. 2002, J. Geophys. Res., 107

Howard, R. A., Sheeley, N. R., Michels, D. J., \& Koomen, M. J. 1985, J. Geophys. Res., 90, 8173

Kliem, B., Titov, V. S., \& Török, T. 2004, A\&A, 413, L23

López Fuentes, M. C., Démoulin, P., Mandrini, C. H., \& van Driel-Gesztelyi, L. 2000, ApJ, 544, 540

López Fuentes, M. C., Démoulin, P., Mandrini, C. H., Pevtsov, A. A., \& van Driel-Gesztelyi, L. 2003, A\&A, 397, 305

Lepping, R. P., Burlaga, L. F., \& Jones, J. A. 1990, J. Geophys. Res., 95,11957

Lin, J., \& Forbes, T. G. 2000, J. Geophys. Res., 105, 2375

Lundquist, S. 1950, Ark. Fys., 2, 361

Manoharan, P. K., van Driel-Gesztelyi, L., Pick, M., \& Démoulin, P. 1996, ApJ, 468, L73

Marubashi, K. 1997, in Coronal Mass Ejections, Geophysical Monograph, 99, 147

Mikic, Z., \& Linker, J. A. 1994, ApJ, 430, 898

Munro, R. H., \& Sime, D. G. 1985, Sol. Phys., 97, 191

Nindos, A., \& Zhang, H. 2002, ApJ, 573, L133

Nishio, M., Nakajima, H., Enome, S., et al. 1994, in Proc. Kofu Symp., 19

Pevtsov, A. A., \& Balasubramaniam, K. S. 2003, Adv. Space Res., 32, 1867 
Pevtsov, A. A., Canfield, R. C., \& Zirin, H. 1996, ApJ, 473, 533

Pevtsov, A. A., Fisher, G. H., Acton, L. W., et al. 2003, ApJ, 598, 1387

Pohjolainen, S. 2000, A\&A, 361, 349

Rust, D. M. 1994, Geophys. Res. Lett., 21, 241

Rust, D. M., \& Kumar, A. 1996, ApJ, 464, L199

Scherrer, P. H., Bogart, R. S., Bush, R. I., et al. 1995, Sol. Phys., 162, 129

Schrijver, C., \& Zwaan, C. 2000, in Solar and stellar magnetic activity (Cambridge University Press), Cambridge Astrophysical Ser., 34

Shibata, K., Ishido, Y., Acton, L. W., et al. 1992, PASJ, 44, L173

Shimazu, H., \& Marubashi, K. 2000, J. Geophys. Res., 105, 2365

Siarkowski, M., Gburek, S., \& Rudawy, P. 2002, Adv. Space Res., 30, 589

Sonnerup, B. U., \& Cahill, L. J. 1967, J. Geophys. Res., 72, 171

Sterling, A. C., \& Hudson, H. S. 1997, ApJ, 491, L55

Sterling, A. C., Hudson, H. S., Thompson, B. J., \& Zarro, D. M. 2000, ApJ, 532, 628

Török, T., \& Kliem, B. 2003, A\&A, 406, 1043
Török, T., \& Kliem, B. 2004, Publications of the Astronomy Deparment of the Eotvos Lorand University, 14, 165

Thompson, B. J., Cliver, E. W., Nitta, N., Delannée, C., \& Delaboudinière, J. P. 2000, Geophys. Res. Lett., 27, 1431

Titov, V. S., \& Démoulin, P. 1999, A\&A, 351, 707

Tsuneta, S., Acton, L., Bruner, M., et al. 1991, Sol. Phys., 136, 37

van-Driel Gesztelyi, 1., Démoulin, P., \& Mandrini, C. H. 2003, Adv. Space Res., 32, 1855

van Driel-Gesztelyi, L., Manoharan, P. K., Démoulin, P., et al. 2000, Journal of Atmospheric and Terrestrial Physics, 62, 1437

Vršnak, B., \& Gopalswamy, N. 2002, Journal of Geophysical Research (Space Physics), 107 (A2), 1019

Yurchyshyn, V. B., Wang, H., Goode, P. R., \& Deng, Y. 2001, ApJ, 563,381

Zarro, D. M., Sterling, A. C., Thompson, B. J., Hudson, H. S., \& Nitta, N. 1999, ApJ, 520, L139

Zhao, X. P., Hoeksema, J. T., \& Marubashi, K. 2001, J. Geophys. Res., 106, 15643 